\title{
1 Resolving tricky nodes in the tree of life through amino acid recoding
}

3 Mattia Giacomelli ${ }^{1}$, Maria Eleonora Rossi ${ }^{2}$, Jesus Lozano-Fernandez ${ }^{3}$, Roberto Feuda ${ }^{4}$,

4 Davide Pisani ${ }^{* 1,2}$

$6{ }^{1}$ School of Biological Sciences, University of Bristol, Life Sciences Building, Tyndall Avenue,

7 Bristol, BS8 1TQ UK

$8{ }^{2}$ School of Earth Sciences, University of Bristol, Life Sciences Building, Tyndall Avenue, Bristol

$9 \quad B S 81 T Q, U K$

$10{ }^{3}$ Department of Genetics, Microbiology and Statistics, Faculty of Biology, University of

11 Barcelona, Spain

$12{ }^{4}$ Department of Genetics and Genome Biology, University of Leicester, Leicester, UK

$14{ }^{*}$ Corresponding author:

15 Davide Pisani: davide.pisani@bristol.ac.uk

\section{Abstract}

19 Genomic data allowed for a detailed resolution of the tree of life. Yet, tricky nodes such as the

20 root of the animal, plants, eukaryotes, bacterial and archaeal trees remain unresolved.

21 Genomic datasets are heterogeneous as genes and species evolve under different selective

22 pressures, impending the efficacy of evolutionary analyses. Amino acid recodings were

23 developed to reduce heterogeneity, but clear evidence to justify their use is missing. We use

24 simulated genomic-scale datasets and show that recodings can substantially improve

25 phylogenetic accuracy when tackling tricky nodes. We apply our findings to address the root

26 of the animal tree where the debate centers on whether sponges (Porifera) or comb jellies

27 (Ctenophora) branched out first. We show that results from real data follow predictions from

28 simulated data and indicate that a placement of the ctenophores as the first branching animal 
29 lineage is most likely artifactual.

\section{Introduction}

32 Understanding of the tree of life has enormously increased since genome-scale (i.e.

33 phylogenomic) datasets became available (e.g. (1-4)). Yet, some tricky nodes remain that are

34 proving difficult to resolve, such as the root of the bacterial, archaeal, eukaryotes, plants and

35 animal trees (5-11). These uncertainties hinder understanding of broader evolutionary

36 questions. For example, uncertainty in animal phylogeny, where the debate centers on

37 whether sponges (Porifera) or comb jellies (Ctenophora) are sister to all other animals $(5,12-$

38 19), affects our understanding of the evolution of body plans, with ramifications reaching areas

39 as different as developmental biology and palaeoecology $(20,21)$.

40 One reason for the difficulty in resolving tricky nodes is the heterogeneity of the

41 evolutionary process. Genome-scale datasets are heterogeneous as different genes, sites

42 and species evolve under different constraints (22). Heterogeneity primarily manifests itself in

43 the form of across-site and -lineage rate heterogeneity (i.e. how many amino acid substitutions

44 are observed at different sites or along different lineages), and across-site and -lineage

45 compositional heterogeneity (i.e. which amino acids are observed at different sites and in

46 different taxa). Evolutionary models that can better account for these forms of heterogeneity

47 (23-25) fit the data better. Yet, even models that can take into consideration different forms of

48 heterogeneity, like the CAT-GTR+G model, which accounts for across-site compositional

49 heterogeneity (the CATegory component (23)), amino acid replacement rate heterogeneity

50 (the General Time Reversible (GTR) matrix (26)) and across-site rate heterogeneity using a

51 Gamma (+G (27)) distribution, frequently fail to fit real world datasets $(4,5)$.

Amino acid recodings were developed to attempt reducing compositional

53 heterogeneity (28-31). These methods (hereafter also referred to as exchangeability-informed

54 recodings, or just recodings), partition the 20 amino acids into bins based on how frequently

55 they exchange with each other (5) - details in Fig.1. Exchangeability-informed recodings are

56 generalizations of standard masking protocols, such as the amino acid recoding of nucleotide 
bioRxiv preprint doi: https://doi.org/10.1101/2022.02.24.479670; this version posted February 24, 2022. The copyright holder for this preprint (which was not certified by peer review) is the author/funder, who has granted bioRxiv a license to display the preprint in perpetuity. It is made available under aCC-BY-NC-ND 4.0 International license.

57 data (32). When exchangeability-informed recodings are used, substitutions among amino

58 acids with similar chemical and physical properties are synonymized (Fig. 1). This is

59 analogous to the process by which codons are synonimized when nucleotides are recoded

60 into amino acids. The rationale underpinning the synonymization procedure is that

61 substitutions among amino acids with similar properties (the within-bin substitutions - Fig. 1)

62 are expected to have a smaller negative impact on the fitness of the coded protein when

63 compared against substitutions among amino acids with different properties (across-bin

64 substitutions - Fig.1, (5)). Within-bin substitutions are thus expected to be more abundant and

65 less constrained, contributing more heterogeneity to the data.

Every masking strategy induces a loss of information $(5,19)$. Yet, amino acid recoding

67 of nucleotide data illustrates that, depending on the distribution of signal and noise, reducing

68 the information content of a dataset can improve the ability of a dataset to resolve phylogenetic

69 relationships (e.g. $(32,33))$. Furthermore, it can be hypothesized that the impact of information

70 loss should decrease as alignment size increases. Nonetheless, it is clear that information

71 loss is potentially a problem when using recodings (19).

Fit is key to phylogenetic accuracy $(25,34-36)$ and it can be relative or absolute (36).

73 Standard model fit tests, such as the Akaike Information Criterion (e.g. (37)), compare the

74 relative fit of different models identifying the one that fits best relative to the others. However,

75 a model of relative best-fit can be a poor descriptor of key properties (e.g. across-site

76 compositional heterogeneity) of the data $(5,36)$. Goodness-of-fit tests (e.g. PPA) evaluate the

77 absolute fit of models to the data (36), and can quantitatively express the extent to which we

78 might be willing to trust results inferred using a model of relative best-fit $(5,25,36,38-40)$.

79 The first attempt at testing the absolute fit of evolutionary models to amino acid and recoded

80 data found that these tests achieve better (i.e. lower) PPA scores on recoded datasets, leading

81 to the suggestion that recoding the data can improve model fit (5). Yet, this argument has

82 been challenged because information loss can also lower PPA scores $(19,41)$.

84 models that account for across-site compositional heterogeneity progressively better. We 
85 tested if the use of recodings can cause a deterioration of the phylogenetic signal, and whether

86 their efficacy depends on alignment size. We tested whether recodings can induce the

87 emergence of tree reconstruction artifacts and devised a new (recoding-based) strategy to

88 test if clades are artifactual. We found that recodings substantially increase accuracy if the

89 model used poorly fits the amino acid data, and the alignment is sufficiently long. If the model

90 fits the amino acid data well, recoding did not improve fit nor accuracy, mildly decreasing

91 support for key nodes instead. However, we find no evidence that the use of recodings might

92 by itself cause the emergence of incorrect but statistically supported relationships (i.e.

93 recodings are conservative). Our results are consistent with the hypothesis that recodings

94 improve fit and consequently accuracy by reducing heterogeneity (5). Accordingly, recodings

95 can only be beneficial when the model does not fit the amino acid data. We propose a guiding

96 principle to decide when recodings can be useful, and confirm that many real datasets might

97 benefit from recoding (5). We test our new approach to identify artifactual clades using the

98 data of (17) to exemplify our approach while tackling one of the trickies nodes in the tree of

99 life, the root of the animal phylogeny $(5-7,12-19,42,43)$. In so doing we found evidence that

100 a placement of the ctenophores at the root of the animal tree is most likely artifactual.

102 Results

\section{Simulated data and their heterogeneity}

104 To avoid experimenter-induced biases, we did not simulate our own datasets. Instead we used

105 the across-site compositional and rate heterogeneous alignments of (18), where the target

106 (true) tree under which the data were generated assumed either that the ctenophores 107 (Ctenophora-sister topology) or the sponges (Porifera-sister topology) are the sister of all the 108 other animals. For each topology, this dataset includes 100 alignments of 30,000 sites and 97 109 taxa, see Methods. The alignments were subsampled to generate datasets of 1,000, 5,000, 11010,000 and 30,000 sites and 20 taxa, as well as datasets with 30,000 sites and 4 or 10 taxa 111 see Supplementary Information (SI), Fig. S1, for the target trees. The alignments were 112 recoded using the Dayhoff-6 amino acid recoding (described in Fig. 1). Unless otherwise 
bioRxiv preprint doi: https://doi.org/10.1101/2022.02.24.479670; this version posted February 24, 2022. The copyright holder for this preprint (which was not certified by peer review) is the author/funder, who has granted bioRxiv a license to display the preprint in perpetuity. It is made available under aCC-BY-NC-ND 4.0 International license.

113 stated, analyses were performed using the 30,000 sites and 20 taxa alignments, under both

114 target topologies, see the flow chart of our experimental design in Fig. S2.

We used PPA of site-specific amino acid diversity (PPA-Div, (44)) to test whether the across-site compositional heterogeneity of the simulated amino acid alignments was comparable to that of real data. These results (reported in $\mathrm{SI}$ ) confirmed that real and simulated data have comparable across-site compositional heterogeneity (Fig. S3), validating

119 the use of the simulated alignments of (18) in our study.

Dayhoff-6 improves accuracy if the model poorly describes the compositional heterogeneity of the amino acid data

We compared the success of amino acid and Dayhoff- 6 recoded data under a series of models allowing for increasing amounts of across-site compositional heterogeneity. Throughout the study we define successful (see methods) an analysis correctly resolving the target topology with a minimal support, measured as its Posterior Probability (PP), of 0.5 (PP $\geq 0.5)$. We

127 distinguish two types of unsuccessful analyses, those that are incorrect (wrong tree inferred with $\mathrm{PP} \geq 0.5$ ), and those that are unresolved (correct or incorrect tree inferred with $\mathrm{PP}<0.5$ ). We performed a sensitivity analysis where we increased the threshold for success to $\mathrm{PP}=$ 0.95. This test did not affect our conclusions, see below and SI for details.

We started from a CAT-based model with 10 frequency categories (nCAT10 - to account for across-site compositional heterogeneity), which was combined with a Poisson 133 process (F81, (45) that does not adjust for replacement rate heterogeneity) and a Gamma 134 distribution $(G)$ modeling across-sites rate heterogeneity. We progressively increased the 135 number of frequency categories to $30,60,120$ and 240 (to accommodate more across-site compositional heterogeneity - analyzing the data using nCAT30-F81+G, nCAT60-F81+G, nCAT120-F81+G and nCAT240-F81+G (hereafter referred to as nCAT10 to nCAT240). In

138 addition, we tested the use of GTR+G (hereafter GTR), which cannot account for across-site 139 compositional heterogeneity but models replacement rate heterogeneity, CAT-F81+G 140 (hereafter CAT) which optimizes the number of categories used to accommodate across-site 
141 compositional heterogeneity during tree search, and CAT-GTR+G (hereafter CAT-GTR) which

142 optimizes the number of categories used and replaces the Poisson process with a GTR matrix

143 to model replacement rate heterogeneity.

144 Previous simulations using amino acid data, showed that it is easier to correctly infer

145 Ctenophora-sister than Porifera-sister (18). While it is not a goal of this study to test why this

146 might be the case, it has been suggested that this could be because Ctenophora-sister lies in

147 the "Farris-zone" (18), having two long branched lineages (outgroups and Ctenophora - Fig.

$148 \mathrm{~S} 1$ ) on one side of the target branch and two short branched lineages (sponges and all the

149 remaining animals) on the opposite side of the target branch (Fig. S1). Our analyses confirmed

150 that Ctenophora-sister is easier to infer than Porifera-sister. Using amino acid data

151 Ctenophora-sister is frequently inferred even when false, if the model has a poor fit (according

152 to PPA) to the data. When Ctenophora-sister is the simulation target it is invariably inferred

153 with a Success Rate (SR) close to $100 \%$, irrespective of model and recoding (see Fig. 2a). On

154 the contrary, Porifera-sister is only retrieved (and not $100 \%$ of the times) when true, with the

155 model and coding strategy used having a strong impact on SR (Fig. 2a). More precisely,

156 Dayhoff-6 increased SR when the model used poorly fit the amino acid data (Figs. 2a-c). To

157 integrate the fact that SRs differ under Porifera- and Ctenophora-sister, for each model and

158 coding scheme we calculated the Total Success Rate (TSR). For each model, this is the

159 percentage of successful analyses, under both target topologies, when amino acids (TSR AA)

160 or Dayhoff-6 (TSR Day $\left._{1}\right)$ are used (Fig. 2b). We observed that the difference between PPA-Div ${ }_{\text {AA }}$

161 and PPA-Div Day (hereafter referred to as dPPA-Div; measured as the difference in Z-score

162 between PPAs performed using differently coded data - see Methods for a guide to interpret

163 Z-score) correlates well $\left(R^{2}=0.90\right)$ with the difference between $T S R_{\text {Day }}$ and $T S R_{A A}$ (hereafter

164 dTSR - Fig. 2d). More precisely, as the evolutionary model better fits the amino acid data

165 dTSR diminishes (Fig. 2d), becoming negative (meaning that TSR $\mathrm{AA}_{\mathrm{AA}}$ was best) for models that

166 fit the amino acid data well (CAT, nCAT240, and CAT-GTR - see Fig. 2c).

167 The CAT-based model with the worst fit to the amino acid data was found to be

168 nCAT10 (Average PPA-DivaA 17; Fig. 2c). Dayhoff-6 data achieved much better PPA-Div 
scores for this model (Average PPADay 2.5), and nCAT10 emerged as the model for which

170 recoding most strongly improved TSR (TSR AA $=57 \%$; TSR $_{\text {Day }}=89.5 \%$; Figs. 2a,b,d - a 32.5\%

171 improvement). On the contrary, the model with the best fit to the amino acid data was CAT-

172 GTR (Average PPA-DivaA $=0.6-$ Fig. 2c). For this model PPA-Div ${ }_{A A}$ and PPA-Div Day were

173 comparable (Average PPA-Div Day -0.9), suggesting that CAT-GTR fits both data types well.

174 CAT-GTR emerged as the model with the worst relative performance under Dayhoff-6

$175\left(\mathrm{TSR}_{\mathrm{AA}}=98 \%\right.$; $\mathrm{TSR}_{\mathrm{Day}}=95 \%$; Fig. $2 \mathrm{~b}$ - corresponding to a 3\% reduction in TSR). Two more

176 models were found to fit the amino acid data well: nCAT240 (Average PPA-Div ${ }_{\mathrm{AA}} \sim 1.5$ ) and

177 CAT (Average PPA-Div ${ }_{A A} \sim 2$ ). As in the case of CAT-GTR, PPA-Divit and PPA-Div Day $_{\text {were }}$

178 comparable for these models, suggesting a good fit to both data types, and as in the case of

179 CAT-GTR, the performance of these models did not improve upon recoding (Figs. 2a and 2d).

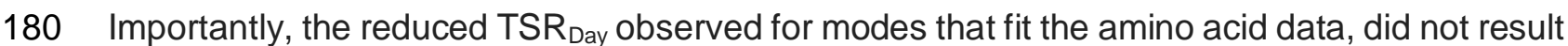

181 from the recovery of incorrect trees, but by an increment in the rate of unresolved outcomes.

182 These results suggest that when the model is already fitting the amino acid data well, we suffer

183 the cost of recoding (information loss), without any benefit (no violation left to palliate). The

184 other CAT-based models tested (nCAT30-nCAT120) had PPA-Div ${ }_{\text {AA }}$ falling within the

185 extremes defined by nCAT10 and CAT (Fig. 2c) and showed intermediate increments in TSR

186 (Figs. 2b and d), which become progressively less marked as PPA-Div $\mathrm{AA}_{\mathrm{A}}$ improved. Finally,

187 GTR performed worse than any of the CAT-based models on both data types, with PPA-Div AA

188 and PPA-Div Day suggesting that this model fit very poorly both types of data (Average PPA-

$189 \operatorname{Div}_{\mathrm{AA}}=43.6$; Average PPA-Div $\left.{ }_{\mathrm{Day}}=30.2\right)$. Consistently with this observation and with the fact

190 that PPA-Div Day potentially indicates a marginally better fit of this model on Dayhoff-6 data,

191 recoding improved the TSR of GTR by $12 \%$, but in a context where, under Porifera-sister, this

192 model was still three times more likely to recover an artifactual Ctenophora-sister topology

193 rather than the target tree.

195 Information loss cannot explain results obtained using Dayhoff-6

196 It has been suggested that the topological changes and reduced PPA scores observed when 
bioRxiv preprint doi: https://doi.org/10.1101/2022.02.24.479670; this version posted February 24, 2022. The copyright holder for this preprint (which was not certified by peer review) is the author/funder, who has granted bioRxiv a license to display the preprint in perpetuity. It is made available under aCC-BY-NC-ND 4.0 International license.

197

198

199

200

201

202

203

204

205

206

207

208

209

210

211

212

213

214

215

216

217

218

amino acid alignments are recoded might be artifacts caused by signal loss (19). We formally tested this hypothesis using a jackknife approach. To achieve our goal, we stripped sites from our amino acid datasets to simulate the effect of a loss of information comparable to that that Dayhoff- 6 would impose if every substitution in the amino acid alignment was phylogenetically informative. This is a worst-case scenario as no dataset is homoplasy-free, and hence the true amount of information that Dayhoff-6 can strip from the data should be lower than the amount we estimate. Accordingly, the results of our test should be conservative and bias our results against Dayhoff-6. For this experiment, datasets were analyzed using nCAT10. This model was chosen because (see Fig.2) it most strongly highlights differences between results obtained using Dayhoff- 6 and amino acids.

The amino acid datasets were found to have an average tree length of $100,914.18$ steps, while the corresponding Dayhoff-6 datasets were found to have an average tree length of $49,683.18$ steps. If we assume each substitution to be phylogenetically informative, we can use tree length as a measure of the phylogenetic signal in the data, and we can estimate that Dayhoff- 6 would reduce the signal in the data by $\sim 49 \%$. We thus jackknifed the amino acids datasets to remove $\sim 49 \%$ of their sites. The average tree length of the jackknifed datasets was $51,179.6$ steps, which is comparable (even if not identical) to the average tree length calculated for the Dayhoff-6 datasets (Fig. 3a), indicating that removing $49 \%$ of the sites from our amino acid datasets generated jackknifed datasets with a number of substitutions comparable to that observed in the Dayhoff-6 datasets. We calculated PPA-Div scores for the jackknifed datasets and compared these values against those achieved by full-length amino acid and Dayhoff-6 datasets. PPA-Div scores for the Jackknifed datasets were significantly worse (Average PPA-Div Jack $=12 ; \mathrm{SD}=0.99$ ) than those of the Dayhoff- 6 datasets (Average PPA-Div ${ }_{\text {Day }}=2.47 ; S D=0.33$ ) and remained comparable to those inferred from full-length amino acid datasets (Average PPA-Div ${ }_{\mathrm{AA}}=17.32 \mathrm{SD}=1.4$; Fig. 3c). The comparison of PPADiv $_{\text {AA }}$ and PPA-Div Jack shows that reducing information indeed mildly reduced PPA-Div scores.

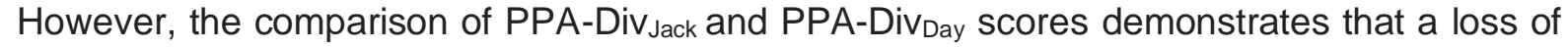
signal comparable to that imposed by the application of Dayhoff-6 cannot explain PPA-Divay 
bioRxiv preprint doi: https://doi.org/10.1101/2022.02.24.479670; this version posted February 24, 2022. The copyright holder for this preprint (which was not certified by peer review) is the author/funder, who has granted bioRxiv a license to display the preprint in perpetuity. It is made available under aCC-BY-NC-ND 4.0 International license. to return non-random trees that will be accurate and well supported if the original amino acid to consider that there is only a limited number of ways in which 20 amino acids can be 252 partitioned across six bins, and random recodings can be expected to retain residual amino

scores, which are significantly better (the distributions do not overlap) than both PPA-DivaA and PPA-Div Jack (Fig. 3c). Furthermore, the TSR of the jackknifed datasets $\left(T_{S R}\right.$ jack $\left.=60.5 \%\right)$ is comparable to that achieved by the full-length amino acid data (TSR $\mathrm{AA}_{\mathrm{A}}=57 \%$ - Fig. S4a) and both are much worse than $\mathrm{TSR}_{\mathrm{Day}}\left(\mathrm{TSR}_{\mathrm{Day}}=89.5 \%\right.$; Fig. $2 \mathrm{~b}$ and $\left.\mathrm{S} 4 \mathrm{a}\right)$. Significantly better $\mathrm{TSR}_{\text {Day }}$ values cannot be caused by a deterioration of the signal in the data, rejecting the hypothesis that results of Dayhoff-6 are driven by signal loss.

\section{Random recodings confirm the efficacy of Dayhoff- 6}

The efficacy of recodings can be investigated by comparing their results to those of randomly generated ones. The latter are recodings where amino acids are randomly reassigned across the bins of an exchangeability-informed (parent) recoding $(19,31)$ - hereafter simply referred to as the "parent".

The theoretical rationale that underpinned the development and application of recodings is grounded on the prediction that there should be more within-bin substitutions than across-bin substitutions (see introduction and Fig.1). If this theoretical prediction is to hold, randomly generated recodings should be expected to mask less substitutions than their parent, and phylogenies inferred from randomly recoded datasets should have higher tree length. In addition, random recodings should not be expected to reduce compositional heterogeneity to the same level of their parent. This is because the masked substitutions, being randomly selected, would not necessarily be between amino acids with similar properties (19). However, it is important to note that random recodings do not randomize the information in the data, they randomly select the subset of substitutions to mask (see Fig. 4). Accordingly, random recodings are Jackknife-like procedures and their application is expected alignment was informative. Nonetheless, the application of random recodings should not be expected to improve accuracy to the same extent of the parent recoding (19). Finally, we need 
acid similarity to their parent.

If the rationale underpinning the use of recodings holds and recodings work as intended, we can thus make three predictions. (I) Phylogenies inferred from randomly recoded datasets will have a higher treelength than those inferred from datasets recoded using the parent recoding. In addition, we expect to observe that the length of trees inferred from randomly recoded datasets will decrease as the residual amino acid similarity between the random recodings and the parent recoding increases. (II) PPA-Div scores of randomly recoded datasets will be worse (higher Z-scores) than those of datasets recoded using the parent recoding. However, we expect Z-scores of randomly recoded datasets to progressively improve as the residual amino acid similarity between the random recodings and their parent increases. (III) The analysis of randomly recoded datasets can be expected to return nonrandom trees, but these trees will be less accurate (lower TSR) than those inferred using the parent recoding. However, the TSR of randomly recoded datasets is expected to improve as the similarity between randomly generated recodings and their parent increases.

We generated 1,000 random recodings (see Methods) based on the Dayhoff-6 binning scheme, finding that they retain on average $36.5 \%$ similarity to Dayhoff-6 (range $0 \%-80 \%$; see 100 recodings of $0 \%$ and $90 \%$ residual amino acid similarity to Dayhoff-6 (hereafter $0 \%$ recodings and 90\%-recodings) using a process where random recodings were generated, compared to their parent, and retained only if they had the desired residual amino acid similarity to Dayhoff-6 (see Methods). We used the 0\%- and 90\%-recodings to test our three predictions.

Our results indicate that the extent to which randomly generated recodings mask substitutions and improve PPA-Div and TSR scores depends on their similarity to Dayhoff-6. $0 \%$-recodings had an average tree length of $88,046.08$ steps and $90 \%$-recodings of $72,390.12$ steps, indicating that Dayhoff-6 silence $\sim 39 \%$ and $\sim 23 \%$ more substitutions than $0 \%$ - and 90\%-recodings (Fig. 3b). PPA-Div scores obtained from Dayhoff-6 (Average PPA-Div Day 2.5; 
$281 \mathrm{SD}=0.33$ ) are significantly better (the distributions do not overlap) than those obtained using

$2820 \%$-recodings (Average PPA-Div $0 \% \sim 6$; SD= 1.2), while the distribution of PPA-Div scores

283 obtained using 90\%-recodings (Average PPA-Div $90 \%$ 4; SD=0.87) overlap with those of both

284 the 0\%-recodings and Dayhoff-6 (Fig. 3d). Finally, we found that the level to which randomly 285 generated recodings improve accuracy $\left(\mathrm{TSR}_{0 \%}=81 \%\right.$; $\left.\mathrm{TSR}_{90 \%}=83.5 \%\right)$ depends on their 286 similarity to Dayhoff-6 (TSR Day $=89.5)$ - Fig. S4b. More precisely, when the target is 287 Ctenophora-sister SR=100\% irrespective of recoding strategy (Figure S4b), while when the 288 target is Porifera-sister $\mathrm{SR}_{0 \%}=62 \%, \mathrm{SR} 90 \%=67 \%, \mathrm{SR}_{\text {Day }}=79 \%$.

\section{The success of Dayhoff-6 increases with the length of the alignment}

291 Recodings mask substitutions and therefore induce a loss of signal. While this loss of signal does not negatively affect results obtained using our 30,000 sites datasets (see above), we hypothesize that the efficacy of recodings should be alignment-length dependent. To test this hypothesis, we analyzed alignments with 20 taxa (Fig. S1) and 1,000, 5,000, 10,000 and 30,000 sites, simulated under both Ctenophora- and Porifera-sister (see above and methods) using nCAT10.

We found that with 1,000 site alignments, amino acids perform better than recodings

$298 \mathrm{TSR}_{\mathrm{AA}}=26 \%$ and $\mathrm{TSR} \mathrm{DAY}_{\mathrm{D}}=11 \%$ (Fig. 5a and Fig. S5). Amino acids perform marginally better 299 than Dayhoff- 6 also with alignments of 5,000 sites $\left(T_{S R} R_{A A}=59.5 \%\right.$ and $T_{S R} R_{\text {Day }}=58.5 \%-$ Fig.

300 S5). However, with alignments of 10,000 sites the overall performance of Dayhoff-6 is better 301 than that of amino acid data $\left(\mathrm{TSR}_{\mathrm{AA}}=63 \%\right.$ and $\mathrm{TSR}_{\mathrm{Day}}=74 \%$; Fig. S5). However, with the 30210,000 site alignments, Dayhoff- 6 does better than amino acids under Porifera-sister $\left(\mathrm{SR}_{\mathrm{AA}}=\right.$ $30326 \%$; SR $\mathrm{AA}_{\mathrm{AA}}=54 \%$ ) while amino acids are still slightly better than Dayhoff-6 under Ctenophora304 sister $\left(\mathrm{SR}_{\mathrm{AA}}=100 \% ; \mathrm{SR} \mathrm{Day}=94 \%\right)$. Finally, with 30,000 site alignments Dayhoff-6 outperforms 305 amino acids $\left(\mathrm{TSR}_{\mathrm{AA}}=57 \%\right.$ and $\mathrm{TSR}_{\mathrm{Day}}=89.5 \%$; Fig. 5b and S5), with Porifera-sister being correctly inferred only $14 \%$ of the times using amino acids and $79 \%$ of the times under Dayhoff6 , when this clade is true. 
bioRxiv preprint doi: https://doi.org/10.1101/2022.02.24.479670; this version posted February 24, 2022. The copyright holder for this preprint (which was not certified by peer review) is the author/funder, who has granted bioRxiv a license to display the preprint in perpetuity. It is made available under aCC-BY-NC-ND 4.0 International license.

309 Sensitivity analyses

310 We tested the effect of our most important experimental choices. We investigated whether the

311 use of Bayesian inference instead of Maximum Likelihood could have affected our results, we

312 studied the effect of changing the number of cycles and runs in our Bayesian analyses, the

313 use of alternative recoding protocols (SR6 and KGB6 - Fig. 1), and the use of a more stringent

314 threshold to define a successful analysis $(P P=0.95)$. We investigated whether taxon sampling

315 density affected our results (subsampling our 30,000 sites datasets to include 10 or 4 taxa),

316 and we tested the effect of combining recodings with an overparameterized CAT-based model

317 that used 544 frequency categories (nCAT544), twice the number that CAT optimally infers

318 on average for our simulated data. The results of these analyses are presented in detail in SI

319 (particularly in Figs. S6 to S11) and show that our results were not affected by our

320 methodological choices. In particular, we found no evidence that combining recodings with an

321 overparameterized model might result in the inference of artifactual trees, as the TSR of

322 nCAT544 remains excellent, irrespective of the coding strategy used $\left(\mathrm{TSR}_{\mathrm{AA}}=96.5 \%\right.$; $T$ SR $\mathrm{R}_{\text {Day }}=$

$32394 \%)$.

324

325

Application of randomly generated recodings to real datasets can be used to identify artifactual clades

327 We applied the knowledge gained from our simulations to a real dataset. We used the dataset 328 chosen by (19), Whelan-strict (17). We analyzed this dataset as an amino acid alignment, as 329 well as using Dayhoff-6, 0\%- and 90\%- recodings using nCAT60, as in (19). We estimated $330 \mathrm{PPA}_{\mathrm{AA}}, \mathrm{PPA}_{0 \%}, \mathrm{PPA}_{90 \%}$, and PPA $\mathrm{Day}_{\text {s. }}$ scores, as well as support values for both Porifera- and 331 Ctenophora-sister under all coding strategies.

Whelan-strict is expected to be affected by both across-site and across-lineage 333 compositional heterogeneity, as it is generally the case for real datasets. We thus used both 334 PPA-Div and PPA-Max (which tests how well a model describes across-lineage compositional 335 heterogeneity (5)) to test the fit of nCAT60 to the data. PPA-Div ${ }_{\text {AA }}$ indicates that nCAT60 336 describes the across-site compositional heterogeneity of Whelan-Strict poorly (PPA-Div $\mathrm{AA}^{\sim}$ 
337 19). PPA-Max indicates that nCAT60 describes relatively well the across-lineage

338 compositional heterogeneity of Whelan-strict (PPA-Max $\mathrm{AA}^{\sim} 2.65$ ), and this form of

339 heterogeneity is thus not expected to represent a major problem for this dataset under

340 nCAT60. The PPA-Div score obtained for Whelan-Strict under nCAT60 is comparable to those

341 obtained (for our simulated data) using nCAT10 - the model for which Dayhoff-6 improved

342 TSR the most (Fig. 2b). Furthermore, PPA-Div Day indicates that while nCAT60 describes the

343 across-site compositional heterogeneity of the amino acid data poorly, it fits well the Dayhoff-

3446 data (PPA-Div $\left.{ }_{\text {Day }}=-0.9\right)$. PPA-Max ${ }_{\text {Day }}=1.91$ indicates that $\mathrm{nCAT60}$ also adequately

345 describes the across-lineage compositional heterogeneity of the Dayhoff-6 data. As expected,

346 values for PPA-Div $0 \%$ and PPA-Div $90 \%$ achieved intermediate values (PPA-Div ${ }_{0 \%}$ 5.4; PPA-

$\left.347 \operatorname{Div}_{90 \%} \sim 2.7\right)$. Based on our simulations, these results are interpreted to suggest that there is

348 scope for recodings to improve the accuracy of nCAT60 with this dataset.

We found that support for Ctenophora-sister was maximal when the data were analyzed as amino acids (Fig. 6) and progressively decreased, reaching minimal support when

351 the data were Dayhoff-6 recoded (Fig. 6). Support for Porifera-sister followed the opposite

352 trend, achieving maximal support under Dayhoff-6 (Fig. 6). We plotted the average support

353 values observed under nCAT10 (the model that in our simulations achieved PPA-Div AA Scores

354 comparable to those obtained using nCAT60 for Whelan-Strict) when both Porifera- and

355 Ctenophora-sister are true (Fig. 6). Changes in support values observed for Porifera-sister

356 are comparable to those observed, using simulated data, when Porifera-sister was true.

357 Differently, the pattern observed for Ctenophora-sister disagrees with what observed in

358 simulations where Ctenophora-sister was true (Fig. 6). The pattern of change in support

359 observed for Porifera-sister is in agreement with what (19) suggested we should observe, if

360 recodings were effective at improving phylogenetic accuracy (and our results suggest that

361 they are) and sponges were the sister of all the other animals. Differently, the pattern of

362 change in support values observed for Ctenophora-sister is consistent with what should be

363 observed if Ctenophora-sister was a tree reconstruction artifact (19). 


\section{Discussion}

\section{The use of recodings is justified}

367 The use of recodings have been theoretically justified based on their predicted ability to mask

368 substitutions that are a-priori expected to be more likely to contribute to the compositional

369 heterogeneity of a data (the within-bin substitutions; e.g. (5)). However, (19) correctly pointed

370 out that there is no published empirical evidence to suggest that the use of recodings is

371 justified. Our random recoding experiments in conjunction with our amino acid jackknife

372 experiment show that: (1) within-Dayhoff-6-bin substitutions are more abundant than across-

373 Dayhoff-6-bin substitutions as Dayhoff-6 remove more substitutions than random recodings;

374 (2) within-bin substitutions contribute more to the compositional heterogeneity of amino acid

375 datasets than across-bin substitutions as Dayhoff-6 improves PPA-Div scores more than

376 random recodings; (3) Dayhoff-6 is effective at improving fit as Dayhoff-6 PPA-DIV scores are

377 significantly lower than those obtained using jackknifed and randomly recoded datasets; (4)

378 Dayhoff-6 improves phylogenetic accuracy as it achieve higher TSR than randomly generated

379 recodings and jackknifed amino acid datasets. Overall, these results indicate that the theory

380 underpinning the use of recodings holds. In addition, these results are consistent with the

381 hypothesis that recodings improve accuracy because, by reducing compositional

382 heterogeneity, they can improve the fit of the model to the data (5). In particular, an improved

383 TSR cannot be explained by a deteriorating signal, and this result is the strongest evidence

384 for the utility of recodings when tackling tricky nodes. The tests of (19) failed to reach these

385 conclusions because it used only four random recodings and did not consider that they

386 retained spurious amino acid similarity (10\% to 40\%) to their parent (SR6).

Notably, even 0\%-recodings outperformed amino acids (compare Figs 2, 3 and S4). It

388 might seem counterintuitive that $0 \%$-recodings can achieve greater TSR (TSR $\%=81 \%)$ than

389 amino acids (TSR $\mathrm{AA}^{\sim}$ 57\%) and it is not the scope of this paper to investigate this

390 phenomenon. However, it should not surprise that 0\%-recodings can infer good quality trees

391 given that randomly recoded datasets are not randomized, they are jackknifed. 
393 Recodings improve accuracy if the model used is not a good descriptor of the

394 compositional heterogeneity of the amino acid data and the alignment is sufficiently

395 long.

396 The relative performance of recoded and amino acid datasets had not been previously

397 investigated using genome scale datasets and models that accommodate compositional

398 heterogeneity progressively better. We show that when phylogenomic-scale alignments are

399 used recodings improve accuracy if the model used had a poor fit to the amino acid data. This

400 might be erroneously interpreted to mean that recodings are not useful if the data are analyzed

401 under the best fit model. That is not the case, as model-fit tests compare the relative fit of

402 models against each other (36), and it has been shown (e.g. (5) and this paper) that relative

403 best fit models can fail absolute goodness-of-fit tests. When these conditions apply (in our

404 study these are the cases of GTR, nCAT10, nCAT30, nCAT60 and nCAT120) phylogenetic

405 accuracy can be improved recoding the data (Fig. 2). On the other hand, when the model fits

406 well the amino acid data (in our study nCAT240, CAT and CAT-GTR) recoding does not

407 improve accuracy. These observations are consistent with the hypothesis that recodings

408 improve accuracy when they allow models to achieve a better fit to the data (5), and can thus

409 only be useful when the model does not already fit the amino acid data well.

410 Recoding data masks substitutions and induces a loss of information. A recent

411 simulation study (41) used alignments of 1,000 to 5,000 sites and concluded that recodings

412 have an invariably deleterious effect on accuracy, challenging more than 100 papers that

413 implemented such approaches. Our results similarly found that with 1,000 and 5,000 sites

414 alignments, amino acids perform better than recodings. However, our results also show that

415 the performance of the recoded data improves as alignment size increases, and with

416 phylogenomic-scale (30,000 site alignments) datasets, Dayhoff-6 clearly outperforms amino

417 acids (when the model used did not fit the amino acid data well - which was the case also in

418 (41)). Our results reject the conclusion of (41), but also indicate, as we predicted, that the

419 efficacy of recodings is alignment size dependent, and their application should be limited to

420 phylogenomic analyses such as those in $(5,6,8,10,16,17)$ and in this study. 


\section{A criterion to decide when to use recodings}

423 Recodings improve accuracy only when their application allows improving fit, and a criterion

424 is needed to decide when they should be used $(7,41)$. We show that the difference in the 425 goodness-of-fit of the model to the amino acid and recoded data ( $\mathrm{PPPA}$ ) correlates strongly $426\left(R^{2}=0.90\right)$ with the difference between the total success rate of recoded and amino acid data 427 (dTSR - Fig. 2). Although it is not clear, on formal grounds, whether PPA-DivaA and PPA428 Div $_{\text {Day }}$ values should be comparable since they are summary statistics computed on data 429 defined on different state spaces, the trend in Figs. $2 \mathrm{~d}$ suggests that these values are 430 nevertheless able to predict if phylogenetic success can be improved using recodings. It 431 should be noted that even if we did not directly compare these values, high PPA-Div scores 432 would still indicate that the model has a lower probability of accurately resolving a phylogeny, 433 compared to a model with a low PPA-Div score. Accordingly, an independent reading of PPA434 Div $_{\text {AA }}$ and PPA-Div Day values (see Table 1), would still allow deciding whether a dataset is best 435 analyzed as an amino acid or a Dayhoff-6 alignment. For example, following the indications 436 in Table 1, one would conclude that under GTR there is a low chance of correctly resolving 437 the target topology irrespective of recoding. Under nCAT10 we would conclude that there is a 438 high probability of correctly resolving the target topology using the recoded data but not the 439 amino acid data, and under CAT-GTR, both data types have a high chance of correctly 440 recovering the target topology.

441 A fundamental aspect of our conclusions is that recodings do not work best with a 442 specific model: that is, our results do not suggest that Dayhoff-6 is best combined with 443 nCAT10. Rather, our results suggest that the effectiveness of recodings depends on their 444 ability to improve the fit of the model to the data. Simulated data lack the complexity of real 445 data and PPA $A_{A A}$ of real data (this study and (5)), indicate that models such as nCAT60 and 446 CAT-GTR can fail to fit real amino acid datasets. Accordingly, combining compositionally

447 heterogeneous models with data recodings have the potential to improve fit and phylogenetic 448 accuracy of real datasets. 


\section{Ctenophora-sister is likely to be a tree reconstruction artifact}

451 Results of posterior predictive analyses (PPA $\left.A_{A A}\right)$ of real datasets assembled to resolve the relationships at the root of the animal tree suggest that these datasets could benefit from recoding (Fig. 6; (5)). We agree with the suggestion of (19) that, if recodings improve accuracy,

454 as indicated by the results of our simulation study, we should be able to use recodings to test

455 if a clade is artifactual or not, as support for artifactual clades should be maximal using amino

456 acid data and minimal using recoded data. Support for true clades should follow the opposite

457 trend, as long as the conditions in which the application of recodings can be useful are met

458 (see above). We should add to the suggestion of (19) that if random recodings were also used,

459 artifactual clades should be expected to lose support as random recodings more similar to 460 their parent are used, while support for true clades should follow the opposite trend. We tested 461 our approach using Whelan-strict (17) under nCAT60, and found that PPA $A$ A and PPA 462 scores suggest that the use of recodings could improve accuracy for this dataset. When 463 plotting support values for Porifera- and Ctenophora-sister using different coding strategies 464 (amino acids, 0\%-, 90\%- and Dayhoff-6 recoding), we found that the trend observed for 465 Porifera sister was that expected for true clades, while the trend observed for Ctenophora466 sister was that expected for tree reconstruction artifacts (Fig. 6). The predictive power of these 467 trends could be confirmed using our simulated data. We thus conclude that our test suggests 468 that Ctenophora-sister is likely to represent a tree reconstruction artifact, and that his new 469 approach can be broadly used to test whether specific resolutions of tricky nodes might 470 represent tree reconstruction artifacts.

$471 \quad$ The debate on the root of the animal tree has seen a diversity of analyses and data.

472 Sponges as the sister of all the other animals is favored by morphology and gene content data 473 (46-48). Amino acid datasets analyzed using CAT and CAT-GTR tend to switch between 474 Porifera- (14) and Ctenophora-sister (49) depending on outgroup sampling. In these 475 experiments Porifera-sister is frequently (but not always) supported when close outgroups, 476 which should reduce attraction artifacts $(5,7,14-17,50)$, are used. A common feature of all 
477 phylogenomic analyses of early animal relationships is that the models used to analyze the

478 amino acid superalignments they use fail to a smaller (with CAT-based models) or larger (with

479 GTR) extent to fit the data (5). When the relevant datasets are recoded, CAT-based models

480 (but not GTR) seem to achieve a good fit ((5) and above). These are the conditions in which

481 we expect that the use of recodings can improve phylogenetic accuracy, and the analysis of recoded data using CAT-based models such as CAT-GTR (or nCAT60 - see above), almost invariably find outgroup-independent support for Porifera-sister - but see (51) for an exception.

Under the interpretative framework of (5), recodings improve model fit and should mitigate attraction artifacts. Accordingly, the broadening of support for Porifera-sister in analyses of recoded datasets was interpreted by (5) as an indication that Ctenophora-sister is

487 a tree reconstruction artifact. Differently, (19) suggested that the results of (5) and of other studies supporting Porifera-sister are either affected by overparameterization-induced artifacts caused by the use of CAT, by biases caused by a deterioration of signal caused by the use of recodings, or, by extension, by a combination of these two factors. studies concluded that overparameterization can reduce support values (decreases precision), but does not cause the emergence of tree reconstruction artifacts, as topological errors disappear as alignment length increases as first noted by (25) and confirmed by (53). We performed our own simulations using a highly overparameterized nCAT544 model, and

496 found, in agreement with $(25,53)$ that it only mildly reduced support values. Similarly, our 497 simulations did not find any evidence that the use of recodings can cause artifacts associated 498 with signal deterioration, not even when Dayhoff-6 is combined with nCAT544. Even in 499 instances where the use of recodings is inappropriate (i.e. when the model already fits the 500 amino acid data), their application only led to mild reductions in support value, indicating that 501 their use is conservative. In particular, we did not find any evidence that Porifera-sister might 502 be inferred as a tree reconstruction artifact, when Ctenophora-sister is the target (19). It has been suggested that we should be skeptical of Porifera-sister because it is found 504 only under a narrow range of models that do not fit the data better than models supporting 
505 Ctenophora-sister (19). However, the size of the model space supporting Ctenophora-sister

506 is not as broad as suggested by (19) because WAG, LG, JTT, etc. (54-56), are just alternative

507 parameterizations of the same model: GTR. If one were to consider alternative

508 parametrizations of CAT as if they were independent models, the range of models under which

509 Porifera-sister is supported will also expand. Furthermore, none of these alternatively

510 parametrized GTRs fits as well as CAT or CAT-GTR (which also frequently support Porifera-

511 sister, e.g. $(14,57)$, contrary to what seems to be implied by (19)). Indeed, (19) only showed

512 that nCAT60 (another CAT-based across-site compositionally heterogeneous model) could

513 not be distinguished in their Bayesian cross-validation from CAT. However, this result does

514 not imply that CAT is overparameterized, as suggested by (19), without performing tests to

515 validate their assertion. Certainly, CAT uses more frequency categories than nCAT60, but the

516 advantage of Bayesian methods is that they allow safely implementing (53) more realistic

517 parameter rich models (23), that can be expected to fit to the data better. Indeed, goodness-

518 of-fit tests show that, irrespective of its status as a relative best fit model, nCAT60 fits Whelan-

519 strict $\left(P P A-D i_{n C A T 60}=18.9\right)$ very poorly, with CAT doing much better $\left(P P A-\right.$ Div $\left._{\text {CAT }}=4.13\right)$, a

520 predictable result.

521 It is our opinion that the solution to the root of the animal tree is not going to be found

522 through a comparison of the number of models (or analyses) supporting Porifera- or

523 Ctenophora-sister. The problem we must solve is why the relative best fit model for the

524 relevant datasets (be that CAT, CAT-GTR, or nCAT60), switch support between Porifera- and

525 Ctenophora-sister as factors such as outgroup sampling or coding strategy are changed. Our

526 hypothesis is that instability under the relative best fit model follows from the fact that also

527 these across-site compositional heterogeneous models struggle to adequately describe these

528 datasets in their amino acid form (5). In such situations recodings can improve fit and 529 accuracy, and recoded analyses performed under CAT, CAT-GTR and nCAT60 (see above), 530 in most cases, reject Ctenophora-sister. We can only conclude that, at the least at time of 531 writing, Ctenophora-sister is only clearly supported by analyses of amino acid datasets 532 performed using models that fail to fit the data, a dubious line of evidence. 


\section{Conclusions}

536 Recodings have been widely applied to investigate some of the trickiest nodes in the tree of

537 life, from the root of the animal (5-7) to that of plants (8), Bacteria (10), and Archaea (9), with

538 more than 100 papers recently published that used these methods as a line of evidence (41).

539 Here we show, using simulations, that recodings are indeed an effective tool to improve

540 phylogenetic accuracy when models do not fit the amino acid data. While a model that does

541 not have a good fit to the data may still infer a correct tree, our simulations show that accuracy

542 significantly improves when models that fit the data well are used. Real datasets assembled

543 to resolve tricky nodes in the tree of life usually display high levels of compositional

544 heterogeneity, and generally fail to be adequately described using available compositionally-

545 heterogeneous models (5). While the development of models that could adequately fit arbitrary

546 levels of across-site and across-lineage compositional heterogeneity would be welcome. It is

547 clear that such models are not yet on the horizon - but see (50) for some interesting recent

548 progress. Until such models are developed the analysis of recoded data will remain a useful

549 line of evidence in the analyses of the heterogeneous datasets generally associated with tricky

550 nodes in the tree of life.

\section{Material and Methods}

553 We tested the efficacy of exchangeability-informed recodings using simulations and real data,

554 performing a variety of tests that are summarized in Fig. S2.

556 The simulated data

557 We used the simulated dataset of (18), where the target tree used to generate the data 558 assumes either Ctenophora-sister or Porifera-sister to be true (Fig. S1). For each target 559 topology, the 100 (30,000 sites and 97 taxa) datasets of (18) were evolved under CAT-LG+G, 560 an across-site rate and compositional heterogeneous model. The original alignments were 
561 subsampled to generate datasets with different numbers of characters and taxa. We

562 generated datasets of $1,000,5,000,10,000$ and 30,000 sites and 20 taxa; as well as datasets

563 of 30,000 sites with 4 and 20 taxa. The number of taxa and characters used in our datasets

564 was chosen to achieve multiple goals. First, we wanted to test the use of recodings using 565 genomic-scale datasets (our 30,000 sites) comparable to those used in real studies. In

566 addition, we wanted to maximize comparability with (41) that used 20 taxa and 1,000 to 5,000

567 sites alignments. Furthermore, we wanted to test the effect of alignment size $(1,000$ to 30,000

568 sites), and taxon sampling density (4 to 20-taxon datasets). When subsampling characters

569 from the datasets of (18), we made sure that each character was sampled only once in each

570 dataset, and we retained taxa in such a way as to continue to include all key lineages

571 necessary to define the two target topologies (see Fig. S1). Taxon subsampling was fixed so

572 that all our 20, 10 and 4 taxon datasets include the same terminals (Fig. S1). A comparison

573 between our results and those of (18) indicated that, by subsampling taxa, we made the

574 inference problem harder (58), as success rates for our 30,000 amino acid datasets is lower

575 than that of (18) under the same models. This is an advantage for our study as it allows

576 comparing the success rate of amino acids and Dayhoff-6, in more challenging conditions.

577 The datasets of (18) were generated using CAT-LG+G. Accordingly, they are only affected by

578 across-site compositional heterogeneity. This is not a problem because exchangeability-

579 informed recordings are expected to reduce both across-site and across-lineage

580 compositional heterogeneity, see (5).

582 Comparing simulated and real data: We first investigated whether the across-site

583 compositional heterogeneity in our datasets was comparable to that of real data. This is

584 necessary to understand the extent to which the analysis of the simulated data can inform real

585 world studies. To achieve this goal, we generated 100 datasets of 1,000 characters and 20

586 taxa each, subsampling the dataset of (49). These 1,000 sites datasets were then compared

587 against the 20 taxa and 1,000 sites datasets generated subsampling the simulated datasets

588 of (18) - under Porifera-sister only. PPA of site-specific amino acid diversity (PPA-Div (44)) 
589 was used to test whether the across-site compositional heterogeneity of these datasets could

590 be adequately modeled using GTR+G (referred to as GTR in the main text), a compositionally

591 homogeneous model. Z-scores, the number of standard deviations separating the

592 heterogeneity of the original dataset from the average heterogeneity of a set 100 posterior

593 predictive datasets simulated under the tested model (in this case GTR) were used to measure

594 the ability of GTR to adequately describe the data (5). As GTR is compositionally

595 homogeneous, and cannot accommodate across-site compositional heterogeneity, if the

596 dataset generated subsampling the alignments of (18) are across-site compositionally

597 heterogeneous, we expect that GTR will fail to adequately model them. Furthermore, If the

598 heterogeneity of the simulated data is comparable to that of real world data, Z-scores from

599 these datasets should be comparable to those obtained from datasets subsampled from (49).

600 Z-scores within the interval included between 2 and -2 indicate that the hypothesis that

601 the model fits the data cannot be rejected (5). Z-scores outside this interval could be taken to

602 indicate that the hypothesis that the model fits the data is rejected. However, these boundaries

603 are arbitrary, as the fit of a model achieving a Z-score of 1.99 and that of a model achieving a

604 Z-score of 2.1 is essentially the same. We prefer a more relaxed interpretation of Z-scores

605 and instead of discussing models as being rejected or not, we say that when $-2<Z<2$ the

606 model fits the data well, or that it adequately describes the data. When $2<|Z|<5$ we consider

607 the fit to vary from fairly good (values close to 2) to fairly poor (values close to 5). As a rule, it

608 seems safe to assume that when $5<|Z|<10$ the fit can be considered poor, with $|Z|>10$

609 indicating a very poor fit of the model to the data. We acknowledge that our boundaries are

610 arbitrary. However, they are in our opinion conservative.

611 All PPA analyses performed to compare real and simulated data were performed in

612 Phylobayes MPI (59) v1.8 (One run, total number of cycles=1,000; burnin= 500; subsampling

613 frequency=5). .

614

615 Analyses of the simulated datasets: For all our analyses we recoded the data using

616 Dayhoff-6 (60), which was chosen because it is the most widely used exchangeability- 
informed recoding (see Fig. 1 for details).

30,000 sites and 20 taxa datasets (assuming both Porifera and Ctenophora-sister to

619 be true) were analyzed under a variety of models $\mathrm{GTR}+\mathrm{G}, \mathrm{nCAT10}-\mathrm{F} 81+\mathrm{G}, \mathrm{nCAT30}-\mathrm{F} 81+\mathrm{G}$, nCAT60-F81+G, nCAT120-F81+G, nCAT240-F81+G, CAT-F81 and CAT-GTR+G (also referred to as GTR and nCAT10 to CAT-GTR in the main text). 1,000 to 10,000 sites datasets were analyzed under nCAT10 only, to test whether the performance of recodings is alignmentsize dependent. 30,000 sites and 20 taxa datasets were analyzed using all the above-

624 mentioned models. Datasets of 4 and 10 taxa and 30,000 sites were analyzed using nCAT10 625 only to test whether the performance of recodings depends on the density of taxon sampling.

626 The model used for the analyses using 1,000 to 10,000 site alignments and 4 and 10 taxon 627 alignments (nCAT10) was selected a posteriori (see main text), as it emerged from the 628 analyses of 30,000 sites and 20 taxa, that nCAT10 maximizes our ability to discriminate between results of Dayhoff-6 and amino acid analyses with our simulated alignments.

All Bayesian analyses were performed in Phylobayes MPI (59) v.1.8 (total number of 631 cycles=2,000; burnin=1,000; subsampling frequency $=10$ ). For each dataset a single run was 632 completed to limit the computational burden and minimize pollution, as customary, see (15, 19). However, to make sure that 2,000 cycles were enough, for the nCAT10 analyses, we performed two more independent runs using 10,000 cycles, testing congruence after 2,000 and 10,000 cycles.

An analysis was deemed successful if it recovered the target tree with a PP $\geq 0.5$. To

637 make sure that using a $\mathrm{PP}=0.5$ cutoff to define success did not bias our results, under 638 nCAT10, we also tested the effect of defining a success using a much more stringent $\mathrm{PP}=$ 6390.95 threshold. In addition, ML analyses were performed in RAXML (61) under GTR for the 64030,000 sites and 20 taxa datasets, with 200 bootstrap replicates, to make sure that using Bayesian analysis did not bias our conclusions. For the 30,000 sites datasets, PPA-Div were completed, for each considered model

643 (total number of cycles $=2,000$; burnin $=1,000$; subsampling frequency $=10$ ). These tests were 644 performed for both the amino acid and the Dayhoff-6 recoded data in Phylobayes (under 
645 Porifera-sister only). We performed some tests (not shown) to make sure that PPA-Div results

646 did not change using chains from the analyses performed with datasets assuming

647 Ctenophora-sister to be true and found that the results did not change. Z-scores (see above)

648 were used to report the results of all PPAs.

650 Amino acid jackknife experiment: To test whether the results of analyses performed using

651 Dayhoff-6 might be driven by an information loss induced when the data are recoded, we

652 performed an experiment where we assumed that every substitution in a dataset is

653 phylogenetically informative. This is an overestimate of the true phylogenetic signal of a

654 dataset (see main text). The experiment represents a worst-case scenario because in a 655 situation of this type, every substitution masked by Dayhoff-6 will reduce phylogenetic signal.

656 If all substitutions in a dataset were phylogenetically informative the phylogenetic signal of a

657 dataset could be estimated as the tree length for the considered dataset. We thus estimated

658 the tree length of our amino acid and Dayhoff-6 datasets and calculated the percent of 659 substitutions observed in the amino acid dataset that are masked by Dayhoff-6 - this turned 660 out to be $49.23 \%$. We randomly stripped $49.23 \%$ of the sites in our amino acid datasets (to 661 simulate a loss of information comparable to that that Dayhoff-6 would induce in our worstcase scenario). The tree length of the $49.23 \%$ jackknifed datasets was re-estimated to quantify whether the number of substitutions in these datasets was now comparable to that in Dayhoff-

6646 datasets. The results showed that the jackknifed datasets and Dayhoff-6 datasets had a 665 comparable number of substitutions. We then proceeded to analyze the jackknifed datasets 666 under nCAT10, estimating PPA-Div values, TSR and comparing these values against those 667 obtained under Dayhoff-6, and for the full-length amino acid datasets. All parsimony analyses 668 were performed using PAUPv4.168 (62) - Parsimony trees were inferred from 1,000 heuristic 669 searches with the multree option turned off. Tree lengths were then estimated using the 670 Pscore command. Parsimony analyses used the 30,000 sites and 20 taxa datasets.

672 Randomly generated recodings experiment: A perl script (Recodings_generator.pl 
673 available: https://bitbucket.org/bzxdp/giacomelliet_al_2022_recodings/src/master/) was

674 written to randomly generate recodings based on the Dayhoff-6 amino acid partitioning

675 scheme: one 5-state bin, two 4-state bins, two 3-state bins and one 1-state bin. This script

676 was used to randomly generate 1,000 recodings. The similarity of these recodings to Dayhoff-

6776 was then estimated using a second script (Recodings_comparator.pl available:

678 https://bitbucket.org/bzxdp/giacomelli_et_al_2022_recodings/src/master/). The latter uses

679 intersections of sets to estimate the percent similarity between bins in randomly generated

680 recodings and their exchangeability-informed parent recoding. The same script was used to

681 compare the four randomly generated recodings of (19) to SR6 (31), their parent

682 exchangeability-informed recoding.

683 We randomly generated 100 recodings of $0 \%$ similarity to Dayhoff- 6 (0\%-recodings),

684 and 100 recodings of $90 \%$ similarity to Dayhoff-6 (90\%-recodings). To do this, we first

685 generated $20,000,000$ recodings using Recodings_generator.pl. After that, we used

686 Recodings_comparator.pl to identify 100 recodings of specified similarity to Dayhoff-6.

Each $(30,000$ sites and 20 taxa) dataset was randomly associated to one of the

688 randomly generated $0 \%$ - and $90 \%$-recodings. We estimated the tree length for the randomly

689 recoded (0\% and 90\%) datasets using PAUPv4.168 (same settings as above). The tree

690 lengths of these randomly recoded datasets were compared against those of amino acid and

691 Dayhoff-6 recoded datasets to test whether Dayhoff-6 silences more substitutions than

692 randomly generated recodings (see main text). After that PPA-Div scores and TSRs were

693 calculated (under nCAT10) for the 0\%- and 90\%-recoded datasets and compared against

694 those obtained using amino acids and Dayhoff-6 (see main text).

695

696 Analysis of real data (Whelan-Strict): We applied our approaches to Whelan-strict (17), the

697 dataset constituting the main focus of (19). This dataset was analyzed as an amino acid

698 alignment and as a 0\%-recoded, 90\%-recoded and Dayhoff-6 recoded dataset, under the

699 model preferred by (19), nCTA60. PPA-Div and PPA-Max values were estimated for each

700 recoding. PPA-Max (14) evaluates whether a model can describe the maximal compositional 
bioRxiv preprint doi: https://doi.org/10.1101/2022.02.24.479670; this version posted February 24, 2022. The copyright holder for this preprint (which was not certified by peer review) is the author/funder, who has granted bioRxiv a license to display the preprint in perpetuity. It is made available under aCC-BY-NC-ND 4.0 International license.

701

702

703

704

705

706

707

708

709

710

711

712

713

714

715

716

717

718

719

720

721

722

723

724

725

726

727

728

729

730

731

732

733

734

735

736

737

738

739

heterogeneity observed across the taxa of a dataset. This test was not used for our simulated data because the model used to simulate the datasets was not across-lineage compositionally

heterogeneous, and thus the simulated alignments were not across-lineage compositionally heterogeneous. However, real datasets are expected to be both across-site and acrosslineage compositionally heterogeneous, and hence the ability of the considered model to describe both forms of heterogeneity need to be tested for Whelan-Strict. Support values obtained for both Porifera-sister and Ctenophora-sister under all recoding strategies considered, were plotted and compared. In addition, we compared these values against the support values (not the SRs) obtained (in our simulations), when Ctenophora-sister and Porifera-sister were true. We plotted the values obtained under nCAT10, because this model achieved PPA-Div scores comparable to that achieved by nCAT60 for Whelan-strict, suggesting a comparable fit of nCAT10 with our simulated data and nCAT60 with WhelanStrict.

\section{References}

1. M. L. Metzker, Sequencing technologies - the next generation. Nat Rev Genet. 11, 3146 (2010).

2. C. W. Dunn, G. Giribet, G. D. Edgecombe, A. Hejnol, Animal Phylogeny and Its Evolutionary Implications. Annu. Rev. Ecol. Evol. Syst. 45, 371-395 (2014).

3. P. Kapli, Z. Yang, M. J. Telford, Phylogenetic tree building in the genomic age. Nat Rev Genet. 21, 428-444 (2020).

4. E. Tihelka, C. Cai, M. Giacomelli, J. Lozano-Fernandez, O. Rota-Stabelli, D. Huang, M. S. Engel, P. C. J. Donoghue, D. Pisani, The evolution of insect biodiversity. Current Biology. 31, R1299-R1311 (2021).

5. R. Feuda, M. Dohrmann, W. Pett, H. Philippe, O. Rota-Stabelli, N. Lartillot, G. Wörheide, D. Pisani, Improved Modeling of Compositional Heterogeneity Supports Sponges as Sister to All Other Animals. Current Biology. 27, 3864-3870.e4 (2017).

6. C. E. Laumer, R. Fernández, S. Lemer, D. Combosch, K. M. Kocot, A. Riesgo, S. C. S. Andrade, W. Sterrer, M. V. Sørensen, G. Giribet, Revisiting metazoan phylogeny with genomic sampling of all phyla. Proc. R. Soc. B. 286, 20190831 (2019).

7. A. K. Redmond, A. McLysaght, Evidence for sponges as sister to all other animals from partitioned phylogenomics with mixture models and recoding. Nat Commun. 12, 1-14 (2021).

8. M. N. Puttick, J. L. Morris, T. A. Williams, C. J. Cox, D. Edwards, P. Kenrick, S. Pressel, C. H. Wellman, H. Schneider, D. Pisani, P. C. J. Donoghue, The Interrelationships of Land Plants and the Nature of the Ancestral Embryophyte. Current Biology. 28, 733745.e2 (2018).

9. T. A. Williams, G. J. Szöllösi, A. Spang, P. G. Foster, S. E. Heaps, B. Boussau, T. J. G. 
bioRxiv preprint doi: https://doi.org/10.1101/2022.02.24.479670; this version posted February 24, 2022. The copyright holder for this preprint (which was not certified by peer review) is the author/funder, who has granted bioRxiv a license to display the preprint in perpetuity. It is made available under aCC-BY-NC-ND 4.0 International license.

Ettema, T. M. Embley, Integrative modeling of gene and genome evolution roots the archaeal tree of life. PNAS. 114, E4602-E4611 (2017).

10. T. A. Williams, C. J. Cox, P. G. Foster, G. J. Szöllösi, T. M. Embley, Phylogenomics provides robust support for a two-domains tree of life. Nat Ecol Evol. 4, 138-147 (2020).

11. R. Derelle, G. Torruella, V. Klimeš, H. Brinkmann, E. Kim, Č. Vlček, B. F. Lang, M. Eliáš, Bacterial proteins pinpoint a single eukaryotic root. Proc Natl Acad Sci USA. 112, E693E699 (2015).

12. C. W. Dunn, A. Hejnol, D. Q. Matus, K. Pang, W. E. Browne, S. A. Smith, E. Seaver, G. W. Rouse, M. Obst, G. D. Edgecombe, M. V. Sørensen, S. H. D. Haddock, A. SchmidtRhaesa, A. Okusu, R. M. Kristensen, W. C. Wheeler, M. Q. Martindale, G. Giribet, Broad phylogenomic sampling improves resolution of the animal tree of life. Nature. 452, 745749 (2008).

13. J. F. Ryan, K. Pang, C. E. Schnitzler, A.-D. Nguyen, R. T. Moreland, D. K. Simmons, B. J. Koch, W. R. Francis, P. Havlak, NISC Comparative Sequencing Program, S. A. Smith, N. H. Putnam, S. H. D. Haddock, C. W. Dunn, T. G. Wolfsberg, J. C. Mullikin, M. Q. Martindale, A. D. Baxevanis, The Genome of the Ctenophore Mnemiopsis leidyi and Its Implications for Cell Type Evolution. Science. 342, 1242592 (2013).

14. D. Pisani, W. Pett, M. Dohrmann, R. Feuda, O. Rota-Stabelli, H. Philippe, N. Lartillot, G. Wörheide, Genomic data do not support comb jellies as the sister group to all other animals. Proc Natl Acad Sci USA. 112, 15402-15407 (2015).

15. P. Simion, H. Philippe, D. Baurain, M. Jager, D. J. Richter, A. Di Franco, B. Roure, N. Satoh, É. Quéinnec, A. Ereskovsky, P. Lapébie, E. Corre, F. Delsuc, N. King, G. Wörheide, M. Manuel, A Large and Consistent Phylogenomic Dataset Supports Sponges as the Sister Group to All Other Animals. Current Biology. 27, 958-967 (2017).

16. N. V. Whelan, K. M. Kocot, L. L. Moroz, K. M. Halanych, Error, signal, and the placement of Ctenophora sister to all other animals. Proc Natl Acad Sci USA. 112, 5773-5778 (2015).

17. N. V. Whelan, K. M. Kocot, T. P. Moroz, K. Mukherjee, P. Williams, G. Paulay, L. L. Moroz, K. M. Halanych, Ctenophore relationships and their placement as the sister group to all other animals. Nat Ecol Evol. 1, 1737-1746 (2017).

18. P. Kapli, M. J. Telford, Topology-dependent asymmetry in systematic errors affects phylogenetic placement of Ctenophora and Xenacoelomorpha. Sci. Adv. 6, eabc5162 (2020).

19. Y. Li, X.-X. Shen, B. Evans, C. W. Dunn, A. Rokas, Rooting the Animal Tree of Life. Molecular Biology and Evolution. 38, 4322-4333 (2021).

20. T. M. Lenton, R. A. Boyle, S. W. Poulton, G. A. Shields-Zhou, N. J. Butterfield, Coevolution of eukaryotes and ocean oxygenation in the Neoproterozoic era. Nature Geosci. 7, 257-265 (2014).

21. D. H. Erwin, The origin of animal body plans: a view from fossil evidence and the regulatory genome. Development. 147, dev182899 (2020).

22. H. Philippe, B. Roure, Difficult phylogenetic questions: more data, maybe; better methods, certainly. BMC Biol. 9, 1-4 (2011).

23. N. Lartillot, H. Philippe, A Bayesian Mixture Model for Across-Site Heterogeneities in the Amino-Acid Replacement Process. Molecular Biology and Evolution. 21, 1095-1109 (2004).

24. P. G. Foster, Modeling Compositional Heterogeneity. Systematic Biology. 53, 485-495 (2004).

25. A. R. Lemmon, E. C. Moriarty, The Importance of Proper Model Assumption in Bayesian Phylogenetics. Systematic Biology. 53, 265-277 (2004).

26. S. Tavaré, in Some mathematical questions in biology DNA sequence analysis (American Mathematical Society, 1986), vol. Vol. 17, pp. 57-86.

27. Z. Yang, Maximum likelihood phylogenetic estimation from DNA sequences with variable rates over sites: Approximate methods. J Mol Evol. 39, 306-314 (1994).

28. M. Embley, M. van der Giezen, D. S. Horner, P. L. Dyal, P. Foster, Mitochondria and hydrogenosomes are two forms of the same fundamental organelle. Phil. Trans. R. Soc. 
bioRxiv preprint doi: https://doi.org/10.1101/2022.02.24.479670; this version posted February 24, 2022. The copyright holder for this preprint (which was not certified by peer review) is the author/funder, who has granted bioRxiv a license to display the preprint in perpetuity. It is made available under aCC-BY-NC-ND 4.0 International license.

Lond. B. 358, 191-203 (2003).

29. I. Hrdy, R. P. Hirt, P. Dolezal, L. Bardonová, P. G. Foster, J. Tachezy, T. Martin Embley, Trichomonas hydrogenosomes contain the NADH dehydrogenase module of mitochondrial complex I. Nature. 432, 618-622 (2004).

30. C. Kosiol, N. Goldman, N. H. Buttimore, A new criterion and method for amino acid classification. Journal of Theoretical Biology. 228, 97-106 (2004).

31. E. Susko, A. J. Roger, On Reduced Amino Acid Alphabets for Phylogenetic Inference. Molecular Biology and Evolution. 24, 2139-2150 (2007).

32. C. E. Laumer, Inferring Ancient Relationships with Genomic Data: A Commentary on Current Practices. Integrative and Comparative Biology. 58, 623-639 (2018).

33. O. Rota-Stabelli, N. Lartillot, H. Philippe, D. Pisani, Serine Codon-Usage Bias in Deep Phylogenomics: Pancrustacean Relationships as a Case Study. Systematic Biology. 62, 121-133 (2013).

34. D. Posada, K. A. Crandall, Selecting the Best-Fit Model of Nucleotide Substitution. Systematic Biology. 50, 580-601 (2001).

35. D. Posada, K. A. Crandall, D. M. Hillis, in Computational and Evolutionary Analysis of HIV Molecular Sequences (Springer US, Boston, MA, 2002), pp. 121-160.

36. D. Shepherd, S. Klaere, How Well Does Your Phylogenetic Model Fit Your Data? Systematic Biology. 68, 157-167 (2019).

37. D. Posada, T. R. Buckley, Model Selection and Model Averaging in Phylogenetics: Advantages of Akaike Information Criterion and Bayesian Approaches Over Likelihood Ratio Tests. Systematic Biology. 53, 793-808 (2004).

38. J. M. Brown, Detection of Implausible Phylogenetic Inferences Using Posterior Predictive Assessment of Model Fit. Systematic Biology. 63, 334-348 (2014).

39. R. Feuda, O. Rota-Stabelli, T. H. Oakley, D. Pisani, The Comb Jelly Opsins and the Origins of Animal Phototransduction. Genome Biology and Evolution. 6, 1964-1971 (2014).

40. J. E. Tarver, M. dos Reis, S. Mirarab, R. J. Moran, S. Parker, J. E. O’Reilly, B. L. King, M. J. O'Connell, R. J. Asher, T. Warnow, K. J. Peterson, P. C. J. Donoghue, D. Pisani, The Interrelationships of Placental Mammals and the Limits of Phylogenetic Inference. Genome Biol Evol. 8, 330-344 (2016).

41. A. M. Hernandez, J. F. Ryan, Six-State Amino Acid Recoding is not an Effective Strategy to Offset Compositional Heterogeneity and Saturation in Phylogenetic Analyses. Systematic Biology. 70, 1200-1212 (2021).

42. H. Philippe, R. Derelle, P. Lopez, K. Pick, C. Borchiellini, N. Boury-Esnault, J. Vacelet, E. Renard, E. Houliston, E. Quéinnec, C. Da Silva, P. Wincker, H. Le Guyader, S. Leys, D. J. Jackson, F. Schreiber, D. Erpenbeck, B. Morgenstern, G. Wörheide, M. Manuel, Phylogenomics Revives Traditional Views on Deep Animal Relationships. Current Biology. 19, 706-712 (2009).

43. T. Nosenko, F. Schreiber, M. Adamska, M. Adamski, M. Eitel, J. Hammel, M. Maldonado, W. E. G. Müller, M. Nickel, B. Schierwater, J. Vacelet, M. Wiens, G. Wörheide, Deep metazoan phylogeny: When different genes tell different stories. Molecular Phylogenetics and Evolution. 67, 223-233 (2013).

44. N. Lartillot, H. Brinkmann, H. Philippe, Suppression of long-branch attraction artefacts in the animal phylogeny using a site-heterogeneous model. BMC Evol Biol. 7, S4 (2007).

45. J. Felsenstein, Evolutionary trees from DNA sequences: A maximum likelihood approach. J Mol Evol. 17, 368-376 (1981).

46. W. Pett, M. Adamski, M. Adamska, W. R. Francis, M. Eitel, D. Pisani, G. Wörheide, The Role of Homology and Orthology in the Phylogenomic Analysis of Metazoan Gene Content. Molecular Biology and Evolution. 36, 643-649 (2019).

47. Y. Zhao, J. Vinther, L. A. Parry, F. Wei, E. Green, D. Pisani, X. Hou, G. D. Edgecombe, P. Cong, Cambrian Sessile, Suspension Feeding Stem-Group Ctenophores and Evolution of the Comb Jelly Body Plan. Current Biology. 29, 1112-1125.e2 (2019).

48. K. Juravel, L. Porras, S. Höhna, D. Pisani, G. Wörheide, "Improved resolution of recalcitrant nodes in the animal phylogeny through the analysis of genome gene content 
bioRxiv preprint doi: https://doi.org/10.1101/2022.02.24.479670; this version posted February 24, 2022. The copyright holder for this preprint (which was not certified by peer review) is the author/funder, who has granted bioRxiv a license to display the preprint in perpetuity. It is made available under aCC-BY-NC-ND 4.0 International license. and morphology" (preprint, Evolutionary Biology, 2021), , doi:10.1101/2021.11.19.469253.

49. E. S. Chang, M. Neuhof, N. D. Rubinstein, A. Diamant, H. Philippe, D. Huchon, P. Cartwright, Genomic insights into the evolutionary origin of Myxozoa within Cnidaria. Proc Natl Acad Sci USA. 112, 14912-14917 (2015).

50. S. A. Muñoz-Gómez, E. Susko, K. Williamson, L. Eme, C. H. Slamovits, D. Moreira, P. López-García, A. J. Roger, Site-and-branch-heterogeneous analyses of an expanded dataset favour mitochondria as sister to known Alphaproteobacteria. Nat Ecol Evol (2022).

51. M. L. Borowiec, E. K. Lee, J. C. Chiu, D. C. Plachetzki, Extracting phylogenetic signal and accounting for bias in whole-genome data sets supports the Ctenophora as sister to remaining Metazoa. BMC Genomics. 16, 1-15 (2015).

52. J. P. Huelsenbeck, B. Rannala, Frequentist Properties of Bayesian Posterior Probabilities of Phylogenetic Trees Under Simple and Complex Substitution Models. Systematic Biology. 53, 904-913 (2004).

53. L. G. Fabreti, S. Höhna, "Bayesian inference of phylogeny is robust to substitution model over-parameterization" (preprint, Evolutionary Biology, 2022), , doi:10.1101/2022.02.17.480861.

54. S. Whelan, N. Goldman, A General Empirical Model of Protein Evolution Derived from Multiple Protein Families Using a Maximum-Likelihood Approach. Molecular Biology and Evolution. 18, 691-699 (2001).

55. S. Q. Le, O. Gascuel, An Improved General Amino Acid Replacement Matrix. Molecular Biology and Evolution. 25, 1307-1320 (2008).

56. D. T. Jones, W. R. Taylor, J. M. Thornton, The rapid generation of mutation data matrices from protein sequences. Bioinformatics. 8, 275-282 (1992).

57. J. T. Cannon, B. C. Vellutini, J. Smith, F. Ronquist, U. Jondelius, A. Hejnol, Xenacoelomorpha is the sister group to Nephrozoa. Nature. 530, 89-93 (2016).

58. D. M. Hillis, Inferring Complex Phylogenies. Nature. 383, 130 (1996).

59. N. Lartillot, N. Rodrigue, D. Stubbs, J. Richer, PhyloBayes MPI: Phylogenetic Reconstruction with Infinite Mixtures of Profiles in a Parallel Environment. Systematic Biology. 62, 611-615 (2013).

60. Dayhoff, M, Schwartz, R., Orcutt, B., in Atlas of protein sequence and structure (1972), p. 5:345-352.

61. A. Stamatakis, RAxML version 8: a tool for phylogenetic analysis and post-analysis of large phylogenies. Bioinformatics. 30, 1312-1313 (2014).

62. D. L. Swofford, "PAUP* ver 4.0. b10." Phylogenetic Analysis Using Parsimony and Other Methods (2003).

\section{Acknowledgments}

We would like to thank Herve Philippe and Gert Wörheide for suggestions on previous iterations of this work. We would also like to thank all the members of the Pisani, Donoghue and Williams labs, at the University of Bristol, for discussing these ideas.

\section{Funding:}

European Union's Horizon 2020 research and innovation program under the Marie Skłodowska-Curie grant agreement (764840) IGNITE (M.G./D.P). 
897 NERC GW4+ Doctoral Training Partnership studentship from the Natural Environment

898 Research Council (S100413) (M.E.R/D.P.).

899 Beatriu de Pinós (Generalitat de Catalunya, 2017-BP-00266) and Juan de la Cierva

900 Incorporación fellowships (Ministerio de Ciencia e Innovación, IJC2018-035237-I) (J.L.F).

901 Royal Society University Research Fellowship (UF160226) (R.F.)

902

903 Author contributions:

904 Conceptualization: D.P., M.G.

905 Methodology: M.G.,D.P.

906 Data curation: M.G.,D.P.

907 Writing-original draft: M.G. D.P.

908 Writing-review \& editing: M.G.,D.P.,M.E.R.,J.L.F.R.F.

909 Supervision:D.P.

910

911 Competing interests:

912 The authors declare that they have no competing interests.

913

914 Data availability:

915 All the data, material and custom scripts used in this study available in the Bitbucket

916 repository: https://bitbucket.org/bzxdp/giacomelli_et_al_2022_recodings/src/master/

918 Figures and Tables

920 Figure 1. The six-bin Dayhoff-6 recoding $(60)$ is the most widely used exchangeability921 informed, and is the one primarily tested in this study. Other recodings include the 6-bin KGB6 922 (30), and SR6 (31), see (5). These recodings differ from Dayhoff-6 in the details of the amino 923 acid partitioning rationale, but the amino acid content of their bins broadly overlap (5). The 924 Dayhoff-6 recoding partitions amino acids into six differently sized bins: one 5-amino acid bin, 
925 two 4-amino acid bins, two 3-amino acid bins and one 1-amino acid bin, based on how

926 frequently they are expected to exchange with each other (i.e. their exchangeability rate). (A)

927 the bins of the Dayhoff-6 recoding and the biochemical properties of the amino acids in each

928 bin. (B) An exemplar amino acid dataset and its Dayhoff-6 recoded representation. Dayhoff-6

929 recoding is achieved by replacing, in a multiple sequence alignment, one letter amino acid

930 codes with one letter codes representing the bins.

932 Figure 2. (A) Success rate of amino acids and Dayhoff-6 as models that can account for more 933 across-sites compositional heterogeneity are used. (B) A table summarizing the Total Success

934 Rate (TSR) for amino acids and Dayhoff-6 under each tested model. TSR is calculated (from 935 the values in Fig. $2 \mathrm{~A}$ ) as the percentage of successful analyses (correct tree inferred with $\mathrm{PP} \geq$ 936 0.5) under both Porifera- and Ctenophora-sister - see also text. (C) Change in the fit of the 937 model to the data (estimated using PPA-Div) as models that can account for more across938 sites compositional heterogeneity are used. In Orange amino acid datasets; in Blue Dayhoff9396 datasets. (D) Correlation between the difference in fit achieved by each considered model 940 on the amino acid and Dayhoff-6 datasets (dPPA-Div), against the difference in TSR achieved 941 before and after recoding ( $(\mathrm{TSSR}$ ). All analyses were run in Phylobayes (Total number of 942 cycles $=2,000$; burnin $=1,000$; subsampling frequency $=10-$ see Sensitivity Analyses for a test 943 of the validity of these settings).

945 Figure 3. (A) Boxplot representing the distribution of tree lengths for the full length amino acid 946 datasets, the 49\% Jackknife amino acid dataset, and the Dayhoff-6 datasets. (B) Boxplot 947 representing the distribution of tree lengths for 0\%-recoded, 90\%-recoded, and Dayhoff-6 948 recoded dataset. (C) PPA-Div scores for the full length amino acid datasets, the $49 \%$ 949 Jackknifed amino acid datasets, and the Dayhoff-6 datasets. (D) Comparison of PPA-Div 950 scores for amino acid datasets, 0\%-recoded, 90\%-recoded, and Dayhoff-6 recoded datasets.

951 The figure indicates that PPA-Div scores of Dayhoff-6 datasets are significantly lower (i.e.

952 better) than PPA-Div scores from jackknifed datasets - panel C, and 0\%-recodings - panel D, 
953 as the distributions do not overlap. PPA-Div refers to analyses performed under nCAT10 in

954 Phylobayes (Total number of cycles=2,000; burnin=1,000; subsampling frequency $=10-$ see

955 Sensitivity Analyses for a test of the validity of these settings).

957 Figure 4. A graphical representation of the similarities between the 0\%-, $90 \%-$ and Dayhoff- 6 recodings of the same amino acid dataset. The alignment represents the first 26 sites of a randomly chosen simulated dataset. The figure shows that there is a clear similarity between

960 the various coding strategies, i.e. random recodings do not scramble the signal in the data;

961 they subsample it. The figure shows that Dayhoff-6 masks more substitutions than the random 962 recodings, indicating that within-Dayhoff-6-bin substitutions are more abundant than across963 Dayhoff-6-bin substitutions. Furthermore, the figure shows that as recodings become more 964 similar to Dayhoff-6 they mask more substitutions (see also Fig. 3 and main text). Note that, 965 in random recodings, amino acids are randomly reassigned to bins, so a common naming 966 system for the bins of different recodings cannot be achieved. Hence in the figure we only 967 use colors. If for a site, all species have the same state (either the same amino acid in the 968 case of the amino acid alignment, or if all amino acids observed at that site fall into the same 969 bin in the case of the recodings), we use a single color to represent the site. If two states are 970 present (either two amino acids or the observed amino acids fall in two different bins) we use 971 two colors. If three amino acids are present or the observed amino acids fall in three bins, we 972 use three colors. The coloring of each column, therefore, simply represents the number of 973 amino acids or bins observed at each position. Across sites, the same color was used for the

974 most common state (irrespective of the bin name for that state), the second most common 975 state, and so on.

977 Figure 5. (A) Success rate of 1,000 sites alignments recoded as amino acid and Dayhoff-6 978 datasets under Ctenophora-sister and Porifera-sister. (B) Success rate of 30,000 sites 979 alignments recoded as amino acid and Dayhoff-6 datasets under Ctenophora-sister and 980 Porifera-sister. All analyses were performed under nCAT10. The figure shows that with 1,000 
bioRxiv preprint doi: https://doi.org/10.1101/2022.02.24.479670; this version posted February 24, 2022. The copyright holder for this preprint (which was not certified by peer review) is the author/funder, who has granted bioRxiv a license to display the preprint in perpetuity. It is made available under aCC-BY-NC-ND 4.0 International license.

981 site alignments amino acids perform better than Dayhoff-6, but with 30,000 site alignments

982 Dayhoff-6 performs better than amino acids. See Figure S5 for the performance of amino acids 983 and Dayhoff-6 with 5,000 and 10,000 site alignments. All analyses were run in Phylobayes

984 (Total number of cycles $=2,000$; burnin $=1,000$; subsampling frequency $=10-$ see Sensitivity 985 Analyses for a test of the validity of these settings).

987 Figure 6. Analyses of Whelan-strict. (A) In Orange: Changes in support for Ctenophora-sister 988 as the data were coded as amino acids, 0\%-, 90\%- and Dayhoff-6 data. Analyses were 989 performed under nCAT60. In Green: Support values obtained in our simulations when 990 Ctenophora-sister is true. The simulated data were analyzed under nCAT10 (see main text and point $\mathrm{C}$ below for the justification for the use of this model). (B) In Orange: Changes in support for Porifera-sister as the data were coded as amino acids, 0\%-, 90\%-, and Dayhoff-6 data. Analyses were performed under nCAT60. In Green: Support values obtained in our simulations when Porifera-sister is true (under nCAT10). (C) Top: PPA-Div scores for the simulated datasets (under nCAT10) when the data are coded as amino acids, 0\%-, 90\%- and Dayhoff-6 data. Bottom: PPA-Div scores for Whelan-strict, under nCAT60, when the data are coded as amino acids, 0\%-, 90\%- and Dayhoff-6 data. Note that analyses were repeated using the complete set of one hundred $0 \%$-recodings and $90 \%$-recodings hence support values and PPA-Div scores for these recodings are average values across 100 analyses.

Table 1. A simple guide to the application of exchangeability-informed recodings. PPA

1002 scores are here intended as absolute values. PPA scores approximately included between 2 1003 and -2 indicate that the model is adequate (Methods for more details).

$1004{ }^{*}$ As a rule of thumb PPA score with an absolute value lower than 2 is considered low. If a 1005 model has a PPA Day score with an absolute value higher than two, it might be worth 1006 considering the use of a model that can accommodate greater amounts of compositional 1007 heterogeneity if available. If such a model is not available our simulations suggest that even 1008 with a PPA ${ }_{\text {Day }}$ score higher than 2, as in the case of nCAT10, an substantial improvement in 
1009 success rate can be observed, if PPA ${ }_{\mathrm{AA}}$ indicated a poor or very poor fit to the data (see

1010 methods for details).

$1011^{* *}$ Note that as PPA $_{A A}$ and PPA Day become closer in value (i.e. as models that can

1012 accommodate greater amount of compositional heterogeneity are used and the fit of the model

1013 to the amino acid data progressively improves) the positive effect of using an exchangeability-

1014 informed recoding decreases (as there are less violations to palliate - see Fig. 2).

$101{ }^{* * *}$ When PPA ${ }_{A A}$ and PPA $A_{\text {Day }}$ scores are high, but PPA $A_{\text {Day }}$ is lower than PPA $A_{A A}$, an improvement

1016 in success rate can still be expected but in the context of an analysis where the chances of

1017 inferring an incorrect tree can still be high (as in the case of GTR).

$1018{ }^{* * *}$ When both PPA $A_{A A}$ and PPA $A_{\text {Day }}$ scores are low (absolute values equal or lower than 2), the

1019 model fits both data types well and the use of recodings cannot be expected to improve fit nor

1020 accuracy, as there are no violations to palliate. 


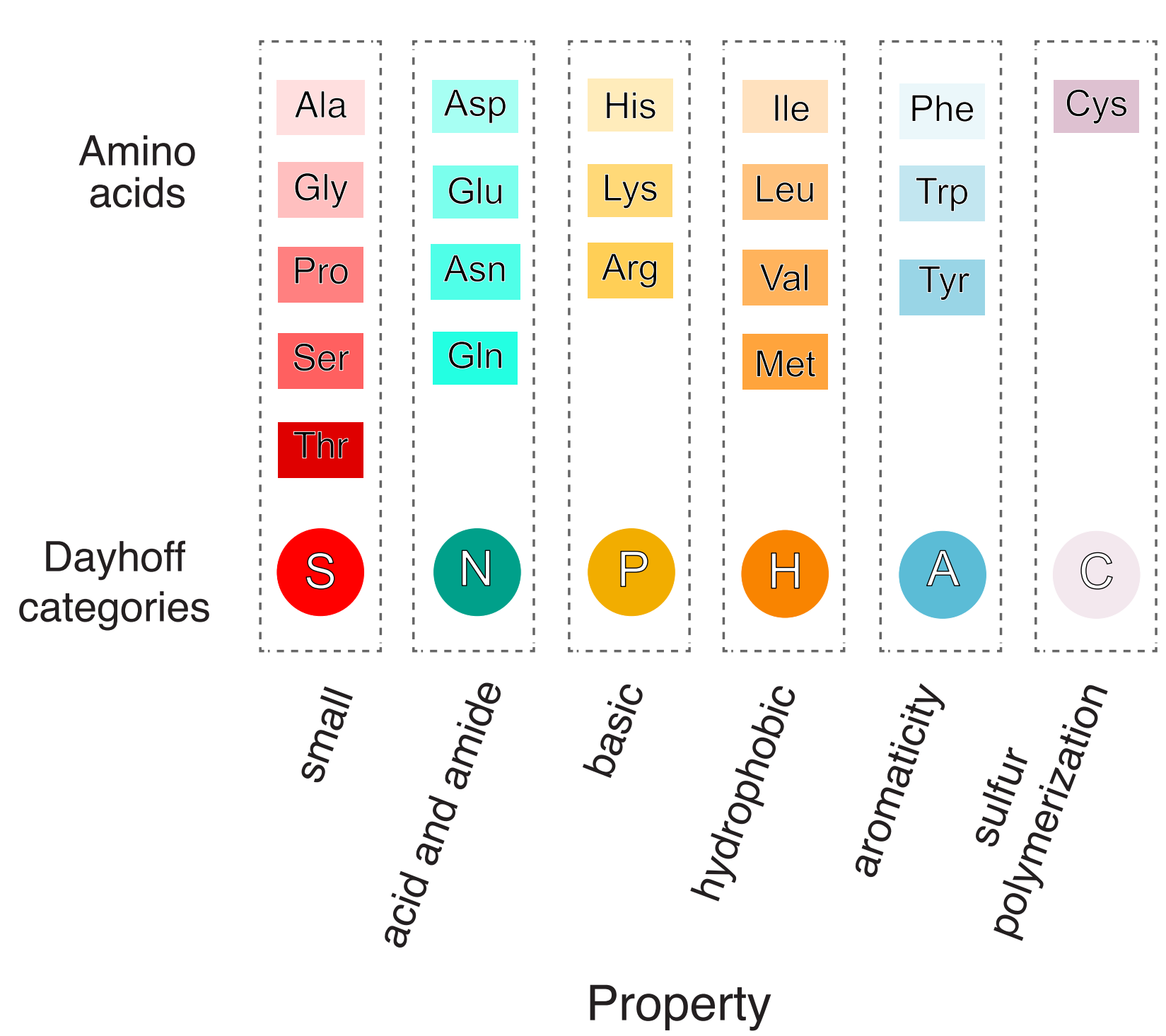

Amino acids

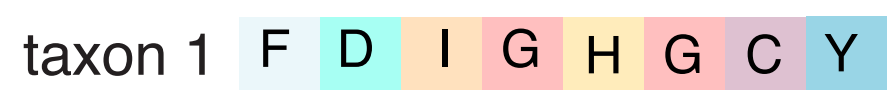

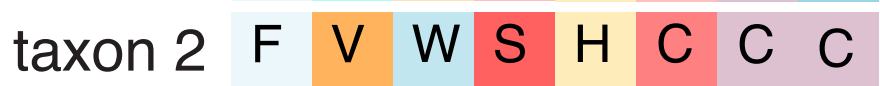
taxon 3 F $N$ N

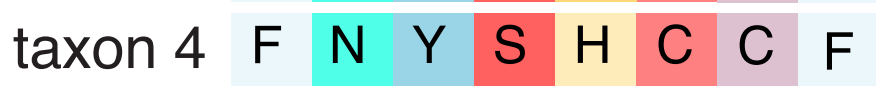

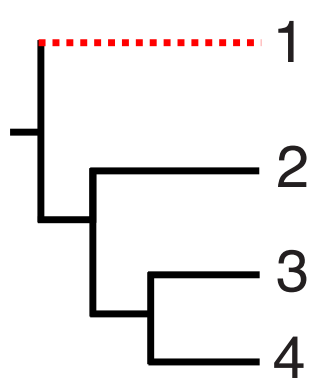

Dayhoff categories

taxon 1 A N N H S P S C A taxon 2 A taxon 3 A $N$ N A S S P $S$ taxon 4 A

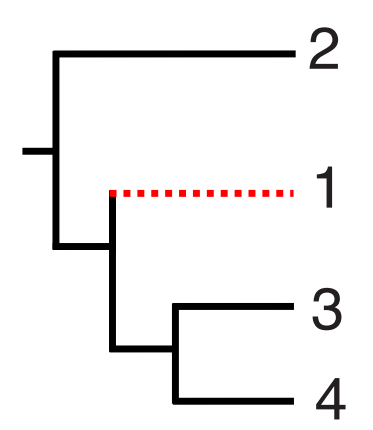

Property 

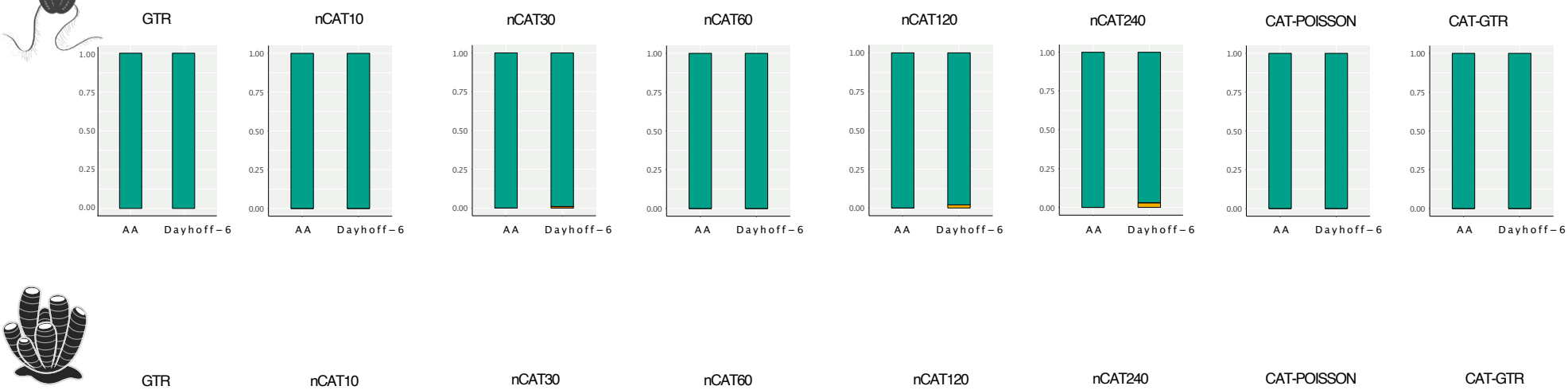

GTR

nCAT10
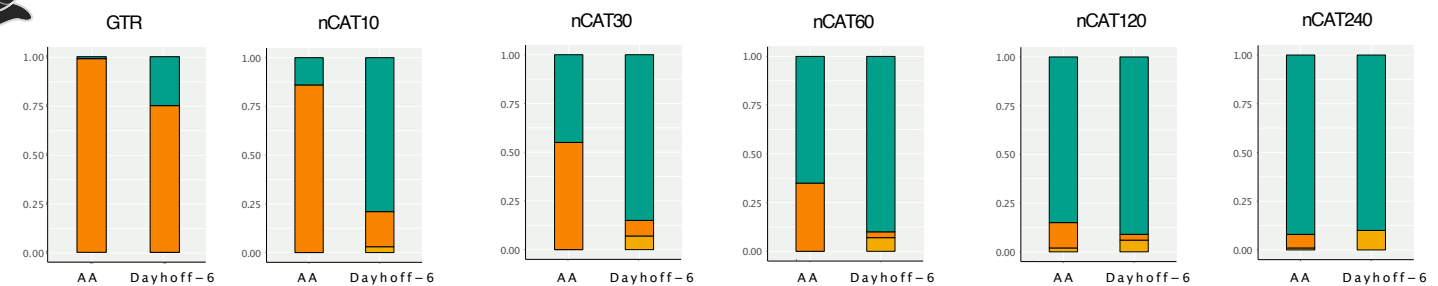

CAT-POISSON

CAT-GTR
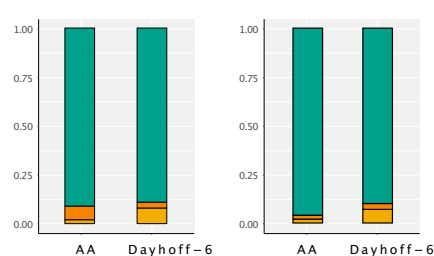

Correct

Incorrect

Uncertain

\section{B}

\begin{tabular}{ccccccccc} 
GTR & nCAT10 & nCAT30 & nCAT60 & nCAT120 & nCAT240 & CAT & CAT+GTR \\
TSR $_{\text {AA }}$ & $50.5 \%$ & $57 \%$ & $72.5 \%$ & $82.5 \%$ & $92.5 \%$ & $96 \%$ & $95.5 \%$ & $98 \%$ \\
\hline TSR $_{\text {Daynoff. }} 62.5 \%$ & $89.5 \%$ & $92 \%$ & $95 \%$ & $94.5 \%$ & $93.5 \%$ & $94.5 \%$ & $95 \%$ \\
& & & & & & & &
\end{tabular}

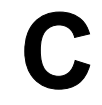

\section{D}
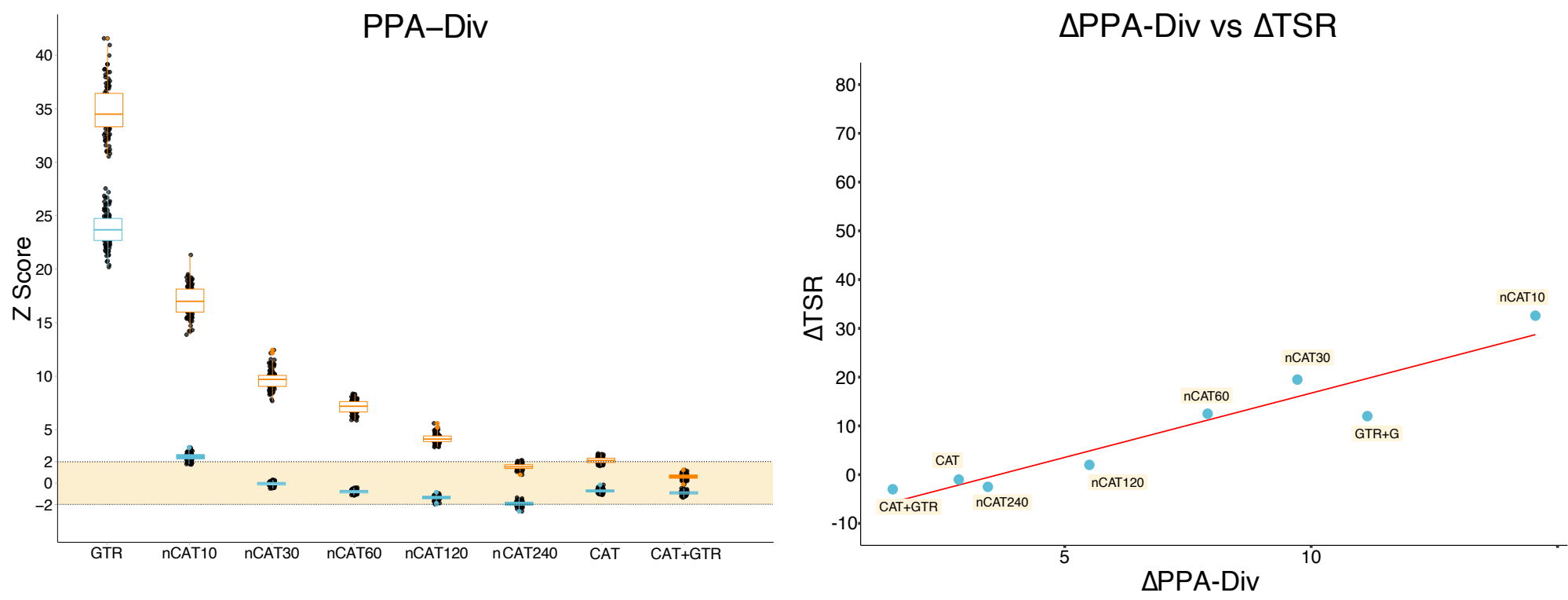

Figure 2 Giacomelli et al. 2022 

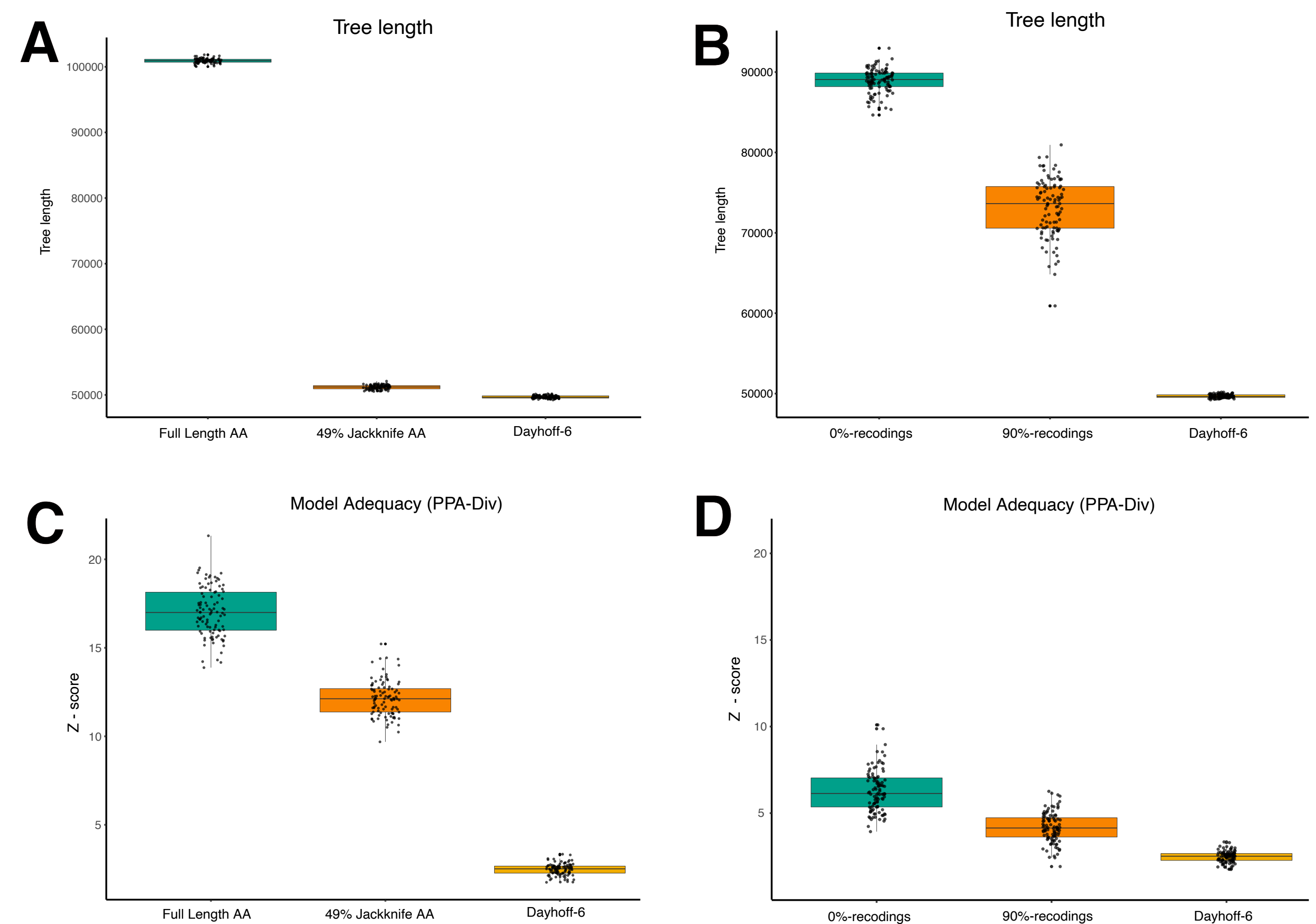

Figure 3 Giacomelli et al. 2022 


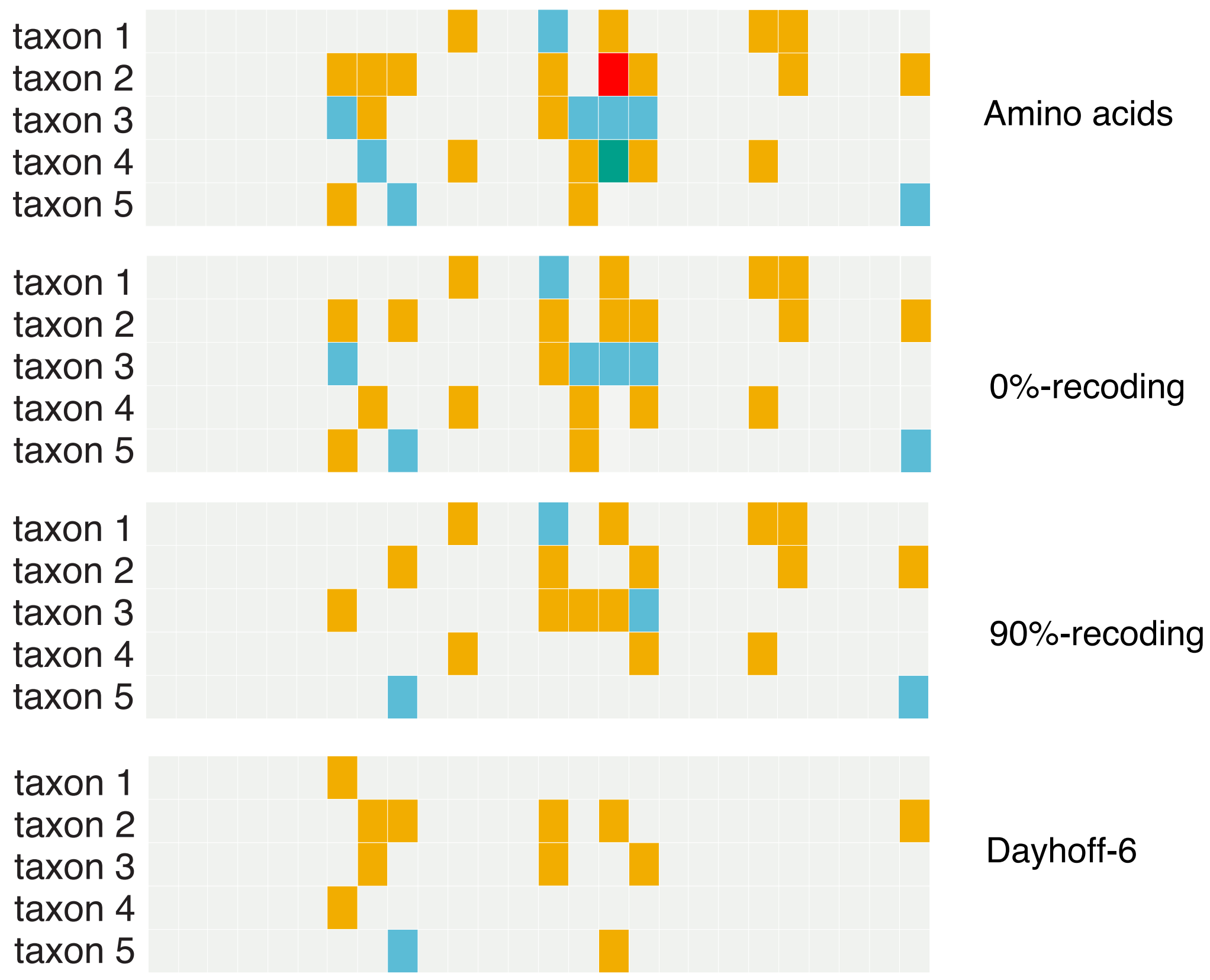


A

B

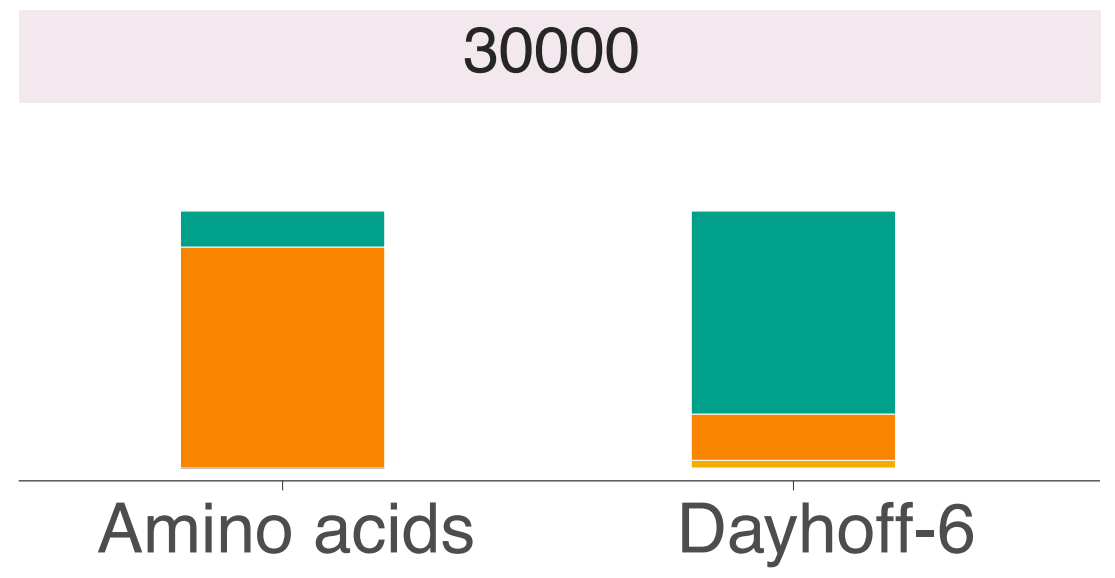

Amino acids Dayhoff-6 
bioRxiv preprint doi: https://doi.org/10.1101/2022.02.24.479670; this version posted February 24, 2022. The copyright holder for this preprint (which was not certified by peer review) is the author/funder, who has granted bioRxiv a license to display the preprint in

A perpetuity. It is made available under aCC-BY-NC-ND 4.0 International license.
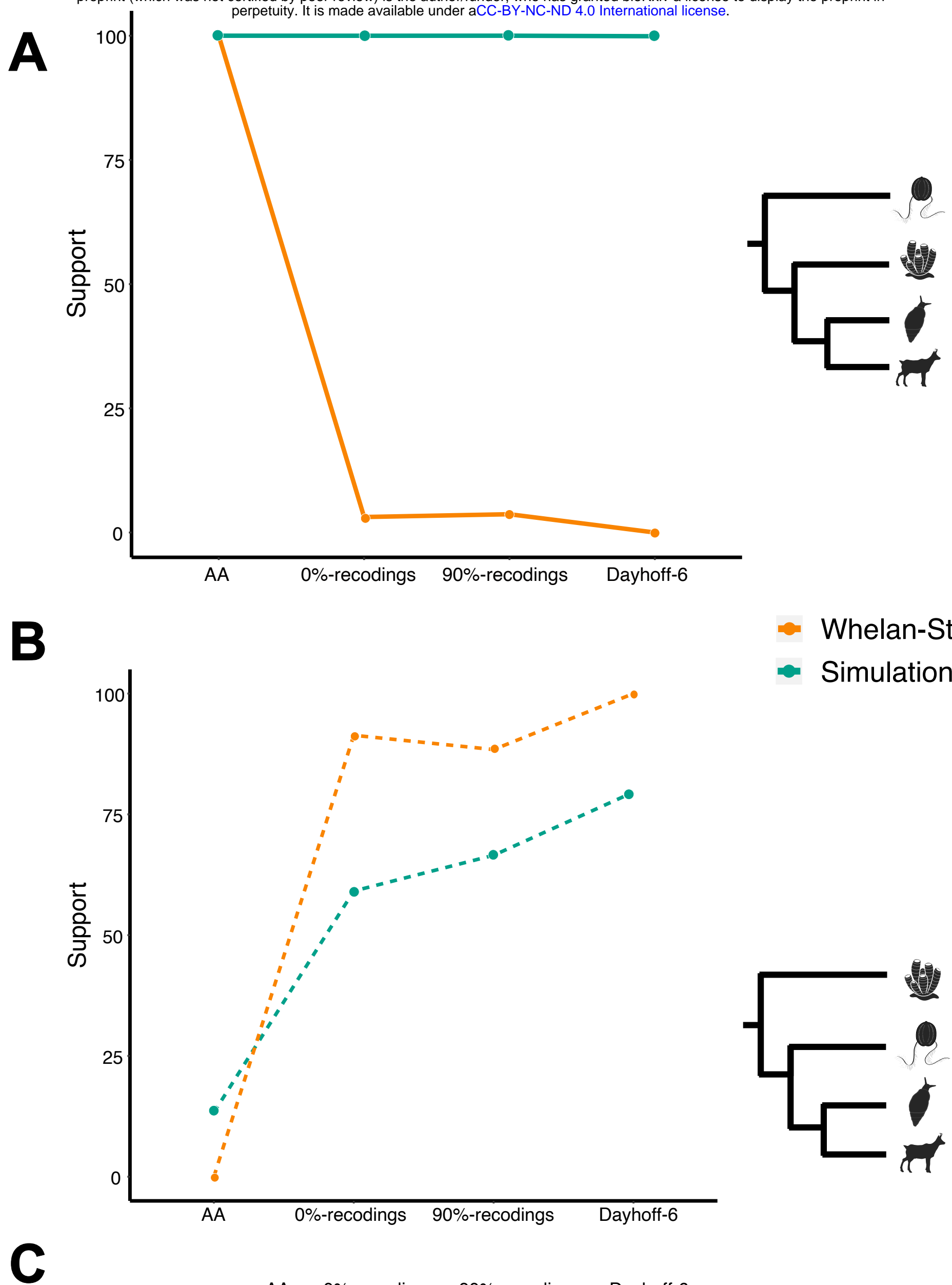

\begin{tabular}{ccccc} 
& AA & 0\%-recodings & 90\%-recodings & Dayhoff-6 \\
\hline Simulations & 17.02 & 6.28 & 4.16 & 2.47 \\
\hline Whelan-Strict & 18.9 & 5.40 & 2.86 & -0.9 \\
\hline
\end{tabular}

Figure 6 Giacomelli et al. 2022 


\begin{tabular}{llll}
\hline $\begin{array}{l}\text { PPAaa score } \\
\text { (absolute value) }\end{array}$ & $\begin{array}{l}\text { PPADay score } \\
\text { (absolute value) }\end{array}$ & $\begin{array}{l}\text { Can recoding improve } \\
\text { accuracy? }\end{array}$ & $\begin{array}{l}\text { Examples from our } \\
\text { simulation study }\end{array}$ \\
\hline High & Yow* & nCAT10 - nCAT120** \\
\hline High & $\begin{array}{l}\text { High }- \text { but still lower } \\
\text { than PPAaa }\end{array}$ & Ges** \\
\hline Low & Low & No & nCAT240, CAT and \\
& & CAT-GTR***
\end{tabular}

